$10 / 121940.80$

SANDIA REPORT

SAND94-1932 • UC-705

Unlimited Release

Printed August 1994

\title{
Stabilizing S.P.H. with Conservative Smoothing
}

\author{
Y. Wen, D. L. Hicks, J. W. Swegle
}

Prepared by

Sandla National Laboratories

Albuquerque, New Mexico 87185 and Livermore, California 94550

for the United States Department of Energy

under Contract DE-ACO4-94AL85000

Approved for public release; distribution is unlimited

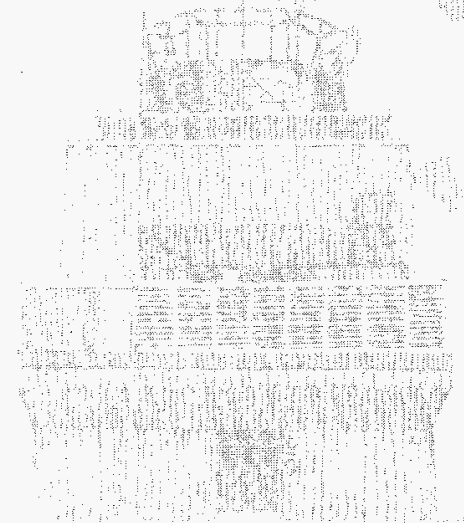


Issued by Sandia National Laboratories, operated for the United States Department of Energy by Sandia Corporation.

NOTICE: This report was prepared as an account of work sponsored by an agency of the United States Government. Neither the United States Government nor any agency thereof, nor any of their employees, nor any of their contractors, subcontractors, or their employees, makes any warranty, express or implied, or assumes any legal liability or responsibility for the accuracy, completeness, or usefulness of any information, apparatus, product, or process disclosed, or represents that its use would not infringe privately owned rights. Reference herein to any specific commercial product, process, or service by trade name, trademark, manufacturer, or otherwise, does not necessarily constitute or imply its endorsement, recommendation, or favoring by the United States Government, any agency thereof or any of their contractors or subcontractors. The views and opinions expressed herein do not necessarily state or reflect those of the United States Government, any agency thereof or any of their contractors.

Printed in the United States of American. This report has been reproduced directly from the best available copy.

Available to DOE and DOE contractors from

Office of Scientific and Technical Information

PO Box 62

Oak Ridge, TN 37831

Prices available from (615) 576-8401, FTS 626-8401

Available to the public from

National Technical Information Service

US Department of Commerce

5285 Port Royal RD

Springfield, VA 22161

NTIS price codes

Printed copy: A04

Microfiche copy: A06 


\section{DISCLAIMER}

Portions of this document may be illegible in electronic image products. Images are produced from the best available original document. 
SAND94-1932

Unlimited Release

Printed August 1994

Distribution

Category UC-705

\title{
Stabilizing S.P.H. with Conservative Smoothing
}

\author{
Y. Wen and D. L. Hicks † \\ Michigan Technological University \\ Houghton, MI 49931 \\ J. W. Swegle $\dagger \dagger$ \\ Solid and Structural Mechanics Department \\ Sandia National Laboratories \\ Albuquerque, New Mexico 87185
}

\begin{abstract}
There is an instability in certain S.P.H. (Smoothed Particle Hydrodynamics method) material dynamics computations. Evidence from analyses and experiments suggests that the instabilities in S.P.H. are not removable with artificial viscosities. However, the analysis shows that a type of conservative smoothing does remove the instability. Also, numerical experiments, on certain test problems, show that SPHCS, an S.P.H. code with conservative smoothing, compares well in accuracy with computations based on the von Neumann-Richtmyer method.
\end{abstract}

$\dagger$ This work was conducted at Michigan Technological University.

$\dagger \dagger$ This work, conducted at Sandia National Laboratories, was supported by the U. S. Department of Energy under Contract No. DE-AC04-94AL85000. 
THIS PAGE INTENTIONALLY LEFT BLANK 


\section{Contents}

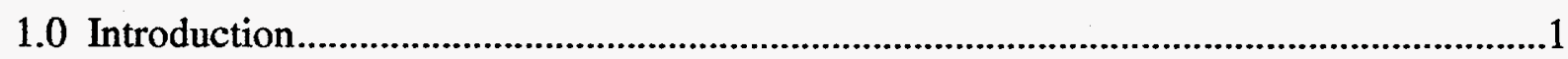

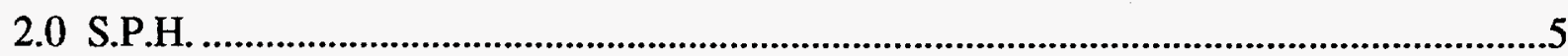

3.0 Stability Analysis of S.P.H. with Artificial Viscosity ............................................

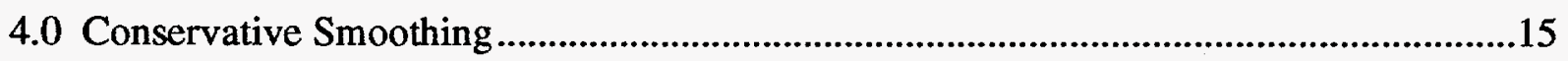

5.0 Stability Analysis of S.P.H. with Conservative Smoothing......................................19

6.0 Results of Computational Experiments .................................................................29

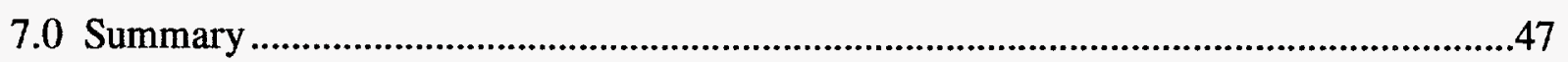

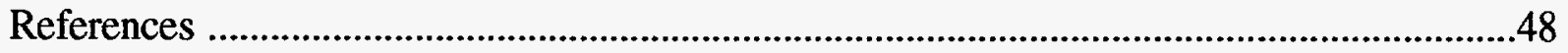




\subsection{INTRODUCTION}

The S.P.H. instability was first documented in [12]. In [8] it was shown that conservative smoothing could stabilize S.P.H. This paper is an extension of the previous analyses. In the previous stability analyses the local equation of first variation (our mathematical model for the propagation of small perturbations in the S.P.H. equations) was developed as a perturbation from a uniform stress state; the following analysis develops the local equation of first variation as a perturbation from a nonuniform stress state.

The von Neumann - Fourier stability analyses involve a Fourier series analysis of the local equation of first variation. Numerous computational experiments with material dynamics codes have shown a close correlation between the predictions of von Neumann - Fourier stability analyses and the actual behavior of computations with respect to discrete - numerical stability. For details on von Neumann - Fourier stability analyses of computational material dynamics methods see [7] and [11].

Let $t^{n}=n \Delta t, \mu_{j}=j \Delta \mu$,

$$
\mathbf{x}_{j}=\left(x_{j-\ell}, \ldots, x_{j-1}, x_{j}, x_{j+1}, \ldots, x_{j+\ell}\right) \in \Re^{2 \ell+1},
$$

and consider the following equation:

$$
{\frac{\Delta^{2} x^{n}}{\Delta t^{2}}}_{j}=F_{j}^{n}\left(\mathbf{x}_{j}^{n-1}, \mathbf{x}_{j}^{n}, \mathbf{x}_{j}^{n+1}\right),
$$

where $F$ is composed of difference operators:

$$
\frac{\Delta}{\Delta t}, \frac{\Delta}{\Delta \mu}, \ldots, \frac{\Delta^{m+r}}{\Delta t^{\tau} \Delta \mu^{m}},
$$

where $\tau=0$ or 1 and $m$ is some nonnegative integer. The equation of first variation of (2) is:

$$
\frac{\Delta^{2} \delta x^{n}}{\Delta t^{2}}{ }_{j}=\delta F_{j}^{n}\left(\mathbf{x}_{j}^{n-1}, \mathbf{x}_{j}^{n}, \mathbf{x}_{j}^{n+1}\right),
$$

where

$$
\delta F_{j}^{n}\left(\mathbf{x}_{j}^{n-1}, \mathbf{x}_{j}^{n}, \mathbf{x}_{j}^{n+1}\right)=\sum_{\nu=-1}^{1} \mathbf{A}^{n, \nu, j} \bullet \delta \mathbf{x}_{j}^{n+\nu}
$$

with

$$
\mathbf{A}^{n, \nu, j}=\operatorname{grad}_{\mathbf{x}_{j}^{n+\nu}} F_{j}^{n}\left(\mathbf{x}_{j}^{n-1}, \mathbf{x}_{j}^{n}, \mathbf{x}_{j}^{n+1}\right) .
$$

Further, $\mathbf{A}^{n, \nu, j}$ is a vector with $2 \ell+1$ components and composed of difference operators as in (3), having coefficients which are functions of

$$
n, j, \Delta t, \Delta \mu, x, \frac{\Delta x}{\Delta t}, \frac{\Delta x}{\Delta \mu}, \ldots, \frac{\Delta^{m+\tau} x}{\Delta t^{\tau} \Delta \mu^{m}}
$$


where $\tau=0$ or 1 and $m$ is some nonnegative integer.

Let $\mathbf{A}_{c}^{n, \nu, j}$ be $\mathbf{A}^{n, \nu, j}$ evaluated at the constant - coefficient data vector, $\mathbf{C}_{c}$, i.e.,

$$
\mathrm{C}_{c}=\left(n_{c}, j_{c}, \Delta t_{c}, \Delta \mu_{c}, x_{c}, \frac{\Delta x}{\Delta t}, \frac{\Delta x}{\Delta \mu_{c}}, \ldots, \frac{\Delta^{m+\tau} x}{\Delta t^{\tau} \Delta \mu^{m}}\right)
$$

where all the components of $\mathbf{C}_{c}$ are constants. Then the equation

$$
\frac{\Delta^{2} \delta x^{n}}{\Delta t^{2}}=\sum_{\nu=-1}^{1} \mathbf{A}^{n, \nu, j} \bullet \delta \mathbf{x}_{j}^{n+\nu}
$$

is called the local (at $\mathbf{C}_{c}$ ) equation of first variation of (2).

For simplicity, assume the material interval is $[0,2 \pi]$, the total number of zones is $J$

$$
J=2 \pi / \Delta \mu
$$

and $J$ is an even integer. Then the shortest wavelength is

$$
\ell_{\min }=2 \Delta \mu
$$

and this is associated with the largest wavenumber

$$
k_{\max }=\pi / \Delta \mu
$$

The set of integers, $k$, such that

$$
-k_{\max } \leq k \leq+k_{\max }
$$

is the set of all possible wave numbers.

We call the following the von Neumann transformation:

$$
F_{v N}\left\langle\delta x_{j}^{n}\right\rangle=\left(E_{k}\right)^{j}\left(\lambda_{k}\right)^{n}
$$

Apply the von Neumann transformation to the difference equation in $\delta x$, i.e., $(9)$, to transform it to the following quadratic equation in $\lambda_{k}$ :

$$
\lambda_{k}^{2}-2 b \lambda_{k}+c=0
$$

with roots

$$
\lambda_{k}^{ \pm}=b \pm\left(b^{2}-c\right)^{1 / 2} .
$$

Recall the solutions to the three - level difference equation

$$
f^{n+1}-2 b f^{n}+c f^{n-1}=0,
$$

are, in the equal roots case, i.e., $b^{2}=c$ and

$$
\lambda_{k}=\underset{2}{\lambda_{k}^{+}}=\lambda_{k}^{-}
$$


of the form

$$
f^{n}=(\alpha+\beta n)\left(\lambda_{k}\right)^{n}
$$

and in the distinct roots case, i.e., $b^{2} \neq c$ and

$$
\lambda_{k}^{+} \neq \lambda_{k}^{-}
$$

of the form

$$
f^{n}=\alpha\left(\lambda_{k}^{+}\right)^{n}+\beta\left(\lambda_{k}^{-}\right)^{n}
$$

The amplification factor for the $k^{t h}$ wavenumber, $r_{k}$, is defined by

$$
r_{k}=\max \left|\lambda_{k}^{ \pm}\right|
$$

Definition: Let $k, \Delta t_{c}, \Delta \mu_{c}, N_{c}$, be fixed finite numbers with further constraints being: wavenumber

$$
k \in\left[-k_{\max },+k_{\max }\right]
$$

temporal increment

$$
\Delta t_{c}>0
$$

material increment

$$
\Delta \mu_{c}>0
$$

total number of timesteps

$$
N_{c} \geq 1
$$

$\mathbf{C}_{c}$ is the constant - coefficient data vector whose components are used in the coefficients of the local equation of first variation, i.e., (9).

In the distinct roots case, i.e.,

$$
\lambda_{k}^{+} \neq \lambda_{k}^{-}
$$

if

$$
\left(r_{k}\right)^{n} \leq 1 \text { for } 1 \leq n \leq N_{c}
$$

or in the equal roots case, i.e.,

$$
\lambda_{k}^{+}=\lambda_{k}^{-},
$$




$$
n\left(r_{k}\right)^{n} \leq 1 \quad \text { for } 1 \leq n \leq N_{c} ;
$$

then we say that (2) is absolutely D.-N. stable at $\left(k, \Delta t_{c}, \Delta \mu_{c}, N_{c}, \mathbf{C}_{c}\right)$.

Definition: If (2) is not absolutely D.-N. stable at $\left(k, \Delta t_{c}, \Delta \mu_{c}, N_{c}, \mathbf{C}_{c}\right)$, then we say that it has an absolutely D.-N. instability or is unstable in the absolute D.-N. sense at $\left(k, \Delta t_{c}, \Delta \mu_{c}, N_{c}, \mathbf{C}_{c}\right)$.

Remark: A necessary and sufficient condition for

$$
n\left(r_{k}\right)^{n} \leq 1
$$

all integer $n \geq 0$ is

$$
0 \leq r_{k} \leq\left(\frac{1}{3}\right)^{\frac{1}{3}} \text {. }
$$




\subsection{S.P.H.}

We present the S.P.H. methodology in five steps. For background on S.P.H. see Barnes-Hernquist(1993).

Step 1. This step involves the Dirac delta distribution [a.k.a., generalized function, see Gel'fand and Shilov (1964, page 1)] centered at $\mathbf{x} \in \Re^{3}$, i.e., $\delta\left(\mathbf{x}_{i}-\mathbf{x}\right.$ ), where $\mathbf{x}_{i}$ is the variable of integration. The Dirac delta identity may be written:

$$
f(\mathbf{x})=\iiint_{\Re^{3}} f\left(\mathbf{x}_{i}\right) \delta\left(\mathbf{x}_{i}-\mathbf{x}\right) d \mathbf{x}_{i} .
$$

Step 2. This step replaces the generalized function, $\delta\left(\mathbf{x}_{i}-\mathbf{x}\right)$, by a true function, $W(\cdot, \cdot)$, called the S.P.H. kernel; $W(\cdot, \cdot)$ is a function of two variables with $\mathbf{x}_{i}-\mathbf{x}$ being the first variable and $h$, called the smoothing length, being the second variable. Replace $\delta\left(\mathbf{x}_{i}-\mathbf{x}\right)$ with $W\left(\mathbf{x}_{i}-\mathbf{x}, h\right)$ to get

$$
f(\mathbf{x}) \approx \iiint_{\Re^{3}} f\left(\mathbf{x}_{i}\right) W\left(\mathbf{x}_{i}-\mathbf{x}, h\right) d \mathbf{x}_{i},
$$

where the ' $\approx$ ' approximation is defined by the

\section{S.P.H. Kernel Constraints:}

SPH1 ( $\delta$ approximator) $[h \rightarrow 0] \Rightarrow[W \rightarrow \delta$ in the generalized function sense $]$;

SPH2 (normalized) $\iiint_{\Re^{3}} W(\mathbf{x}, h) d \mathbf{x}=1$;

SPH3 (compact support) $\|\mathbf{x}\| \geq 2 h \Rightarrow W(\mathbf{x}, h)=0$;

SPH4 (flat top) $\operatorname{grad}_{\mathbf{x}} W(\mathbf{0}, h)=\mathbf{0}$;

SPH5 (smooth) $W(\cdot, h) \in \mathcal{C}^{n}, n \geq 0$;

SPH6 (nonnegative) $\forall \mathbf{x}, W(\mathbf{x}, h) \geq 0$;

SPH7 (radially - symmetric) $\|\mathbf{x}\|=\|\mathbf{y}\| \Rightarrow W(\mathbf{x}, h)=W(\mathbf{y}, h)$;

SPH8 (radially - decreasing) $\|\mathbf{x}\| \geq\|\mathbf{y}\| \Rightarrow W(\mathbf{x}, h) \leq W(\mathbf{y}, h)$.

Remark: Sometimes one or more of these constraints are relaxed.

Step 3. This step substitutes

$$
f(\mathbf{x})=\mathbf{F}(\mathbf{x})
$$

into (34) to get

$$
\mathbf{F}(\mathbf{x}) \approx \iiint_{\Re^{3}} \mathbf{F}\left(\mathbf{x}_{i}\right) W\left(\mathbf{x}_{i}-\mathbf{x}, h\right) d \mathbf{x}_{i}
$$


and then takes the divergence with respect to $\mathrm{x}$ of both sides of (36) to get

$$
\operatorname{div}_{\mathbf{x}} \mathbf{F}(\mathbf{x}) \approx-\iiint_{\Re^{3}} \mathbf{F}\left(\mathbf{x}_{i}\right) \operatorname{grad}_{\mathbf{x}} W\left(\mathbf{x}_{i}-\mathbf{x}, h\right) d \mathbf{x}_{i} .
$$

Remark: Alternatively, the standard, but longer, S.P.H. procedure gets (37) by substituting $d i v_{\mathbf{x}} \mathbf{F}(\mathbf{x})$ into (34), integrating by parts, applying the Gauss divergence theorem, and then using the compact support constraint, i.e., SPH3, to arrive at (37).

Step 4. This step transforms continuum integrals to discrete sums in preparation for digital computation,

$$
\iiint_{\Re^{3}} \mathbf{H}(\mathbf{x}) d \mathbf{x} \doteq \sum_{i=1}^{N_{p}} \mathbf{H}\left(\mathbf{x}_{i}\right) V o l_{i}
$$

where $N_{p}$ is the number of S.P.H. particles, $\mathbf{x}_{i}$ is the spatial coordinate of the $i^{\text {th }}$ particle, $V_{o l}$ is the volume and

$$
V o l_{i}=m_{i} V_{i}=m_{i} / \rho_{i},
$$

where $m_{i}$ is the mass, $V_{i}$ is the specific volume, and $\rho_{i}$ is the mass density of the $i^{t h}$ particle.

We thus approximate (34) as follows

$$
f(\mathbf{x}) \dot{\approx} \sum_{i=1}^{N_{p}} f\left(\mathbf{x}_{i}\right) \dot{W}\left(\mathbf{x}_{i}-\mathbf{x}, h\right) V o_{i}
$$

where $\dot{\approx}$ indicates the composition of the $\approx$ and $\doteq$ approximations. Similarly, $(37)$ may be approximated as follows

$$
d i v_{\mathbf{x}} \mathbf{F}(\mathbf{x}) \dot{\approx}-\sum_{i=1}^{N_{p}} \mathbf{F}\left(\mathbf{x}_{i}\right) g r a d_{\mathbf{x}} W\left(\mathbf{x}_{i}-\mathbf{x}, h\right) \operatorname{Vol}_{i}
$$

Step 5. This step applies the $\dot{\approx}$ approximation to continuum material dynamics equations to produce discrete material dynamics equations. We illustrate on the following momentum evolution equation:

$$
\rho \dot{\mathrm{u}}=-\operatorname{div} \sigma
$$

where $\sigma$ is the Cauchy stress and with the overdot indicating the time derivative at a fixed point, $\mathbf{X}_{j}$, in the material, i.e.,

$$
\left.\dot{\mathbf{u}}_{j} \equiv \frac{\partial \mathbf{u}}{\partial t}\right|_{\mathbf{x}_{j}}
$$

and thus $\dot{\mathbf{u}}_{j}$ is the acceleration of particle $j$. A substitution of $\sigma$ for $\mathbf{F}$ in (41) yields

$$
m_{j} \dot{\mathbf{u}}_{j} \dot{\approx} \sum_{i=1}^{N_{p}} \sigma\left(\mathbf{x}_{i}\right) \operatorname{grad}_{\mathbf{x}} W\left(\mathbf{x}_{i}-\mathbf{x}_{j}, h\right) V o l_{i} V o l_{j}
$$




\subsection{Stability Analysis of S.P.H. with Artificial Viscosity}

The S.P.H. instability occurs in one -, two -, and three - dimensions and as the one - dimensional case is much the simplest we, therefore, present a simple set of one dimensional S.P.H. equations to be analyzed for stability.

Let $X$ be the material - length - coordinate (a.k.a., Lagrange - length - coordinate) and let $\mu$ be the material - mass - coordinate (a.k.a., Lagrange - mass - coordinate) where $\mu$ is defined in terms of $X$ and the initial mass density at $X, \rho^{\circ}(X)$, as follows

$$
\mu=\int_{0}^{X} \rho^{0}\left(X_{i}\right) d X_{i} .
$$

Let $x$ be the spatial - length - coordinate (a.k.a., Euler - length - coordinate) and let $\chi(\cdot, \cdot)$ be the motion function, i.e., if

$$
x_{j}^{n}=\chi\left(\mu_{j}, t^{n}\right),
$$

then at time $t^{n}, x_{j}^{n}$ is the position in the spatial reference frame of the material point labeled $\mu_{j}$. Similarly, the specific momentum, $u_{j}^{n}$, in the spatial reference frame at $\left(\mu_{j}, t^{n}\right)$ is given by

$$
u_{j}^{n}=\partial \chi\left(\mu_{j}, t^{n}\right) / \partial t
$$

and likewise, the specific volume, $V_{j}^{n}$, at $\left(\mu_{j}, t^{n}\right)$ is given by

$$
V_{j}^{n}=\partial \chi\left(\mu_{j}, t^{n}\right) / \partial \mu .
$$

Assume uniform material increments, i.e.,

$$
\mu_{j+1}-\mu_{j}=\Delta \mu=m / \text { Area }
$$

and assume unit cross sectional area, i.e.,

$$
\text { Area }=1 .
$$

Using

$$
V_{j} \Delta \mu=V_{o l}
$$

a one - dimensional S.P.H. equation for the evolution of the specific momentum may be written as

$$
\dot{u}_{j}=\Delta \mu \sum_{i=1}^{N_{p}} V_{i} V_{j} \sigma_{i, j} W_{i, j}^{\prime} \operatorname{sign}\left(x_{i}-x_{j}\right),
$$

where

$$
W_{i, j}^{\prime} \operatorname{sign}\left(x_{i}-x_{j}\right)=\partial W\left(\left|x_{i}-x_{j}\right|\right) / \partial x_{i}
$$


with

$$
\sigma_{i, j}=p_{i}+q_{i, j}
$$

and $q_{i, j}$ is the viscous stress between particles $i$ and $j$.

Single increment difference operators are denoted by an upper case delta with subscripts $\mu$ or $t$, when needed, e.g.,

$$
\Delta_{\mu} x_{j+1 / 2}=x_{j+1}-x_{j}, \quad \Delta_{t} x^{n+1 / 2}=x^{n+1}-x^{n},
$$

but subscripts are suppressed when clear from context, e.g.,

$$
\frac{\Delta x}{\Delta \mu}=\frac{\Delta_{\mu} x}{\Delta \mu} \text { and } \frac{\Delta x}{\Delta t}=\frac{\Delta_{t} x}{\Delta t} .
$$

Similarly, double increment difference operators are denoted by:

$$
D_{\mu} x_{j}=\left(x_{j+1}-x_{j-1}\right) / 2 \text { and } \frac{D x}{\Delta \mu}=\frac{D_{\mu} x}{\Delta \mu} .
$$

If

$$
0<\Delta_{\mu} x \leq 2 h \leq 2 D_{\mu} x
$$

then the sum in $(52)$ reduces to

$$
\dot{u}_{j}=\Delta \mu \sum_{i=j-1}^{j+1} V_{i} V_{j} \sigma_{i, j} W_{i, j}^{\prime} \operatorname{sign}\left(x_{i}-x_{j}\right)
$$

which may be written as

$$
-\frac{\partial u_{j}}{\partial t}=\left.\frac{D P}{\Delta \mu}\right|_{j} \equiv \frac{P_{j, j+1}-P_{j, j-1}}{2 \Delta \mu},
$$

where

$$
P_{j, j \pm 1}=\sigma_{j, j \pm 1} A_{j, j \pm 1}
$$

with

$$
A_{j, j \pm 1}=-2 W_{j, j \pm 1}^{\prime} V_{j \pm 1} V_{j}(\Delta \mu)^{2}
$$

Material law: For purposes of stability analysis it is convenient to introduce the linear $p V$ law:

$$
P_{H}(V)=p_{m c}-a_{m c}^{2}\left(V-V_{m c}\right),
$$

which is a special case of Hooke's Law with $a_{m c}$ being the acoustic impedance; the $m c$ - subscript on a symbol indicates a material constant. This determines the p - part of 
our material law and for the q - part we take a simple, single increment version, of the von Neumann - Richtmyer - Landshoff form

$$
q_{j, j \pm 1}=-\nu a_{m c} \Delta_{\mu} u_{j \pm 1 / 2}
$$

where $\nu \geq 0$ is the dimensionless coefficient of artificial viscosity. Our $t$ - discretization of $(60)$ is 65

$$
\Delta_{t} u_{j}^{n} \equiv u_{j}^{n+1 / 2}-u_{j}^{n-1 / 2}=-\left.\Delta t \frac{D P}{\Delta \mu}\right|_{j} ^{n},
$$

where

$$
u_{j}^{n+1 / 2}=\left.\frac{\Delta x}{\Delta t}\right|_{j} ^{n+1 / 2}
$$

and

$$
\begin{gathered}
P_{j, j \pm 1}^{n}=\sigma_{j, j \pm 1}^{n} A_{j, j \pm 1}^{n}, \quad \sigma_{j, j \pm 1}^{n}=p_{j \pm 1}^{n}+q_{j \pm 1 / 2}^{n-1 / 2}, \\
q_{j \pm 1 / 2}^{n-1 / 2}=-\nu a \Delta_{\mu} u_{j \pm 1 / 2}^{n-1 / 2}, \quad p_{j \pm 1}^{n}=P_{H}\left(V_{j \pm 1}^{n}\right) .
\end{gathered}
$$

Taking the volume of particle $j$ to be

$$
V o l_{j}=D_{\mu} x_{j}
$$

yields

$$
V_{j}=\left.\frac{D x}{\Delta \mu}\right|_{j}
$$

Substitute (66) into (65) to get

$$
\left.\frac{\Delta^{2} x}{\Delta t^{2}}\right|_{j} ^{n}=-\left.\frac{D P(x)}{\Delta \mu}\right|_{j} ^{n}
$$

where

$$
\left.\frac{\Delta^{2} x}{\Delta t^{2}}\right|_{j} ^{n} \equiv \frac{x_{j}^{n+1}-2 x_{j}^{n}+x_{j}^{n-1}}{\Delta t^{2}}
$$

and $P(x)$ means we are going to write $P$ in terms of $x$, i.e., $P(x)=\sigma(x) A(x)$, where

$$
\sigma(x)_{j, j \pm 1}^{n}=P_{H}(0)-a_{m c}^{2} D_{\mu} x_{j \pm 1}^{n} / \Delta \mu-\nu a_{m c} \Delta_{\mu} \Delta_{t} x_{j \pm 1 / 2}^{n-1 / 2} / \Delta t
$$

and

$$
A(x)_{j, j \pm 1}^{n}=-\left.\left.2(\Delta \mu)^{2} W^{\prime}(x)_{j, j \pm 1}^{n} \frac{D x}{\Delta \mu}\right|_{j} ^{n} \frac{D x}{\Delta \mu}\right|_{j \pm 1} ^{n},
$$


further

$$
W^{\prime}(x)_{j, j \pm 1}^{n}=W^{\prime}\left(\Delta_{\mu} x_{j \pm 1 / 2}^{n}, h\right) .
$$

Now we compute the equation of first variation for $(70)$ :

$$
\left.\frac{\Delta^{2} \delta x}{\Delta t^{2}}\right|_{j} ^{n}=-\left.\frac{D \delta[\sigma(x) A(x)]}{\Delta \mu}\right|_{j} ^{n},
$$

where

$$
\delta[\sigma(x) A(x)]=\delta[\sigma(x)] A(x)+\sigma(x) \delta[A(x)]
$$

with

$$
\delta[\sigma(x)]_{j, j \pm 1}^{n}=-a_{m c}^{2} D_{\mu} \delta x_{j \pm 1}^{n} / \Delta \mu-\nu a_{m c} \Delta_{\mu} \Delta_{t} \delta x_{j \pm 1 / 2}^{n-1 / 2} / \Delta t
$$

and

$$
\begin{aligned}
& \delta[A(x)]_{j, j \pm 1}^{n} /\left(-2(\Delta \mu)^{2}\right)=\left.\left.\delta W^{\prime}(x)_{j, j \pm 1}^{n} \frac{D x}{\Delta \mu}\right|_{j} ^{n} \frac{D x}{\Delta \mu}\right|_{j \pm 1} ^{n} \\
& +\left.\left.W^{\prime}(x)_{j, j \pm 1}^{n} \frac{D \delta x}{\Delta \mu}\right|_{j} ^{n} \frac{D x}{\Delta \mu}\right|_{j \pm 1} ^{n}+\left.\left.W^{\prime}(x)_{j, j \pm 1}^{n} \frac{D x}{\Delta \mu}\right|_{j} ^{n} \frac{D \delta x}{\Delta \mu}\right|_{j \pm 1} ^{n},
\end{aligned}
$$

further

$$
\delta W^{\prime}(x)_{j, j \pm 1}^{n}=W^{\prime \prime}(x)_{j, j \pm 1}^{n} \Delta_{\mu} \delta x_{j \pm 1 / 2}^{n}
$$

Notation note: Double increment material averaging is denoted by an overbar; e.g., the double increment material average of $\sigma$ at $\mu_{j}$ is denoted $\bar{\sigma}_{j}$ and given by

$$
\bar{\sigma}_{j}=\left(\sigma_{j, j+1}+\sigma_{j, j-1}\right) / 2 \text {. }
$$

Observe that, by the discrete product rule, i.e., $D(x y)=\bar{x} D(y)+D(x) \bar{y}$, we have

$$
\frac{D \delta[\sigma A]}{\Delta \mu}=\overline{\delta \sigma} \frac{D A}{\Delta \mu}+\frac{D \delta \sigma}{\Delta \mu} \bar{A}+\bar{\sigma} \frac{D \delta A}{\Delta \mu}+\frac{D \sigma}{\Delta \mu} \overline{\delta A}
$$

hence (75) expands to

$$
-\frac{\Delta^{2} \delta x}{\Delta t^{2}}=\overline{\delta \sigma} \frac{D A}{\Delta \mu}+\frac{D \delta \sigma}{\Delta \mu} \bar{A}+\bar{\sigma} \frac{D \delta A}{\Delta \mu}+\frac{D \sigma}{\Delta \mu} \overline{\delta A} .
$$

For simplicity in earlier analyses we chose to perturb from a state of uniform stress, $\sigma$, and uniform $\Delta x$. Here we are going to do a more general stability analysis where we perturb from a nonuniform state of stress but still uniform $\Delta x$. If we perturb from a uniform- $\Delta x$-state, where $\Delta x=\Delta x_{c}$ is constant uniformly in $\mu$, then also uniformly constant are $V=V_{c}, W^{\prime}=W_{c}^{\prime}, W^{\prime \prime}=W_{c}^{\prime \prime}$. Thus

$$
A=A_{c} \quad \text { and } \quad \frac{D A}{\Delta \mu}=0, A=A_{c}
$$


Therefore, (82) reduces to

$$
-\frac{\Delta^{2} \delta x}{\Delta t^{2}}=\frac{D \delta \sigma}{\Delta \mu} A_{c}+\bar{\sigma} \frac{D \delta A}{\Delta \mu}+\frac{D \sigma}{\Delta \mu} \overline{\delta A}
$$

From (77):

$$
{\frac{D \delta \sigma^{n}}{\Delta \mu}}_{j}=-a_{m c}^{2} D_{\mu}^{2} \delta x_{j}^{n} /(\Delta \mu)^{2}-\nu a_{m c} \Delta_{\mu}^{2} \Delta_{t} \delta x_{j}^{n-1 / 2} /(2 \Delta \mu \Delta t) .
$$

From (78) and (79):

$$
\delta A_{j, j \pm 1}^{n} /\left(-2(\Delta \mu)^{2}\right)=V_{c}^{2} W_{c}^{\prime \prime} \Delta_{\mu} \delta x_{j \pm 1 / 2}^{n}+\left.W_{c}^{\prime} V_{c} \frac{D \delta x}{\Delta \mu}\right|_{j} ^{n}+\left.W_{c}^{\prime} V_{c} \frac{D \delta x}{\Delta \mu}\right|_{j \pm 1} ^{n},
$$

therefore,

$$
\overline{\delta A_{j}^{n}} /\left(-2(\Delta \mu)^{2}\right)=V_{c}^{2} W_{c}^{\prime \prime} D_{\mu} \delta x_{j}^{n}+\left.W_{c}^{\prime} V_{c} \frac{D \delta x}{\Delta \mu}\right|_{j} ^{n}+\left.W_{c}^{\prime} V_{c} \frac{D \overline{\delta x}}{\Delta \mu}\right|_{j} ^{n},
$$

and

$$
\frac{D \delta A^{n}}{\Delta \mu}{ }_{j} /\left(-2(\Delta \mu)^{2}\right)=V_{c}^{2} W_{c}^{\prime \prime} \Delta_{\mu}^{2} \delta x_{j}^{n} /(2 \Delta \mu)+\left.W_{c}^{\prime} V_{c} \frac{D^{2} \delta x}{\Delta \mu^{2}}\right|_{j} ^{n} .
$$

Recall that $\nu, a_{m c}, \Delta \mu, \Delta t$ are constants. We emphasize this point: $\Delta \mu$ and $\Delta t$ are fixed, finite constants, as they are in a computer calculation; they should not be thought of as 'arbitrarily small' nor as 'going to zero'. Henceforth, to reduce notational clutter, we suppress the ' $c$ ' subscripts, unless they seem needed for clarity.

Let:

$$
E_{\text {stuff }}=\left(E_{k}\right)^{j}\left(\lambda_{k}\right)^{n}
$$

and apply the $F_{v N}\langle\cdot\rangle$ transformation to get:

$$
F_{v N}\left\langle\left.\frac{D^{2} \delta x}{\Delta \mu^{2}}\right|_{j} ^{n}\right\rangle=E_{s t u f f}\left(E_{k}^{2}-2+E_{k}^{-2}\right) /\left[4(\Delta \mu)^{2}\right]
$$

and:

$$
F_{v N}\left\langle\left.\frac{\Delta^{2} \delta x}{\Delta \mu^{2}}\right|_{j} ^{n}\right\rangle=E_{s t u f f}\left(E_{k}-2+E_{k}^{-1}\right) /(\Delta \mu)^{2}
$$

further:

$$
F_{v N}\left\langle\left.\frac{\Delta^{3} \delta x}{\Delta \mu^{2} \Delta t}\right|_{j} ^{n-1 / 2}\right\rangle=E_{s t u f f}\left(E_{k}-2+E_{k}^{-1}\right)\left(1-\lambda_{k}^{-1}\right) /\left[\Delta t(\Delta \mu)^{2}\right]
$$


furthermore:

$$
F_{v N}\left\langle\left.\frac{D \delta x}{\Delta \mu}\right|_{j} ^{n}\right\rangle=E_{s t u f f}\left(E_{k}-E_{k}^{-1}\right) /(2 \Delta \mu),
$$

moreover:

$$
F_{v N}\left\langle\left.\frac{D \overline{\delta x}}{\Delta \mu}\right|_{j} ^{n}\right\rangle=E_{s t u f f}\left(E_{k}^{2}-E_{k}^{-2}\right) /(4 \Delta \mu),
$$

finally:

$$
F_{v N}\left\langle\left.\frac{\Delta^{2} \delta x}{\Delta t^{2}}\right|_{j} ^{n}\right\rangle=E_{s t u f f}\left(\lambda_{k}-2+\lambda_{k}^{-1}\right) /(\Delta t)^{2}
$$

Cancelling out the $E_{s t u f f}$, the von Neumann-Fourier stability analysis of (84) reduces to

$$
\begin{gathered}
\left(\lambda_{k}-2+\lambda_{k}^{-1}\right) /(\Delta t)^{2}= \\
A\left\{a^{2}\left(E_{k}^{2}-2+E_{k}^{-2}\right) /(2 \Delta \mu)^{2}+\nu a\left(E_{k}-2+E_{k}^{-1}\right)\left(1-\lambda_{k}^{-1}\right) /(2 \Delta t \Delta \mu)\right\} \\
\left.+\bar{\sigma}\left\{\Delta \mu V^{2} W^{\prime \prime}\left(E_{k}-2+E_{k}^{-1}\right)+W^{\prime} V\left(E_{k}^{2}-2+E_{k}^{-2}\right) / 2\right]\right\} \\
+\frac{D \sigma}{\Delta \mu}\left\{2 \Delta \mu\left[\left(\Delta \mu V^{2} W^{\prime \prime}+W^{\prime} V\right)\left(E_{k}-E_{k}^{-1}\right)+W^{\prime} V\left(E_{k}^{2}-E_{k}^{-2}\right) / 2\right]\right\} .
\end{gathered}
$$

Now let $s_{k}=\sin k \Delta \mu$ and $c_{k}=\cos k \Delta \mu$, then Euler's identity, $E_{k}=c_{k}+i s_{k}$, yields the following trigonometric identities:

$$
E_{k}+E_{k}^{-1}=2 c_{k}, \quad E_{k}-E_{k}^{-1}=2 i s_{k},
$$

and:

$$
E_{k}-2+E_{k}^{-1}=2\left(c_{k}-1\right)
$$

further:

$$
E_{k}^{2}-2+E_{k}^{-2}=\left(E_{k}-E_{k}^{-1}\right)^{2}=-4 s_{k}^{2}
$$

furthermore:

$$
E_{k}^{2}-E_{k}^{-2}=\left(E_{k}-E_{k}^{-1}\right)\left(E_{k}+E_{k}^{-1}\right)=4 i c_{k} s_{k} .
$$

Using the foregoing trigonometric identities reduces (96) to:

$$
\begin{gathered}
\lambda_{k}-2+\lambda_{k}^{-1}= \\
A\left\{-s_{k}^{2} C_{F L}^{2}+\nu C_{F L}\left(c_{k}-1\right)\left(1-\lambda_{k}^{-1}\right)\right\} \\
-2(\Delta t)^{2} \bar{\sigma}\left\{\Delta \mu V^{2} W^{\prime \prime}\left(1-c_{k}\right)+W^{\prime} V s_{k}^{2}\right\} \\
+2 i s_{k}(\Delta t)^{2} D_{\mu} \sigma\left\{\Delta \mu V^{2} W^{\prime \prime}+W^{\prime} V\left(1+c_{k}\right)\right\},
\end{gathered}
$$


where $C_{F L}=a \Delta t / \Delta \mu$ is the Courant-Friedrichs-Lewy(1928) number.

Remark: Note that the $D_{\mu} \sigma$-term in (101), i.e., the last term in (101):

$$
2 i s_{k}(\Delta t)^{2} D_{\mu} \sigma\left\{\Delta \mu V^{2} W^{\prime \prime}+W^{\prime} V\left(1+c_{k}\right)\right\}
$$

is the extra term introduced by going from a uniform to a nonuniform stress in the stability analysis. Further, note that this term contains the only imaginary numbers in (101), i.e., all the other terms contain real numbers. Note that in the $\bar{\sigma}$-term in (101), i.e., the second term in (101):

$$
-2(\Delta t)^{2} \bar{\sigma}\left\{\Delta \mu V^{2} W^{\prime \prime}\left(1-c_{k}\right)+W^{\prime} V s_{k}^{2}\right\}
$$

there is a certain product: $\bar{\sigma} W^{\prime \prime}$. This term, i.e.,

$$
\bar{\sigma}_{j} W_{c}^{\prime \prime}=W_{c}^{\prime \prime}\left(\sigma_{j, j+1}+\sigma_{j, j-1}\right) / 2
$$

turns out to be the crucial term in the SPH instability.

Thus the stability polynomial equation is

$$
\lambda_{k}^{2}-2 B \lambda_{k}+C=0
$$

where

$$
C=1-\alpha, \quad \alpha=\nu A C_{F L}\left(1-c_{k}\right),
$$

and

$$
B=1-\Xi_{F L}-\alpha / 2+i \beta
$$

where

$$
\Xi_{F L}=\left\{\left(1+c_{k}\right)\left[A C_{F L}^{2} / 2+\bar{\sigma} W^{\prime} V(\Delta t)^{2}\right]+\bar{\sigma} W^{\prime \prime} V^{2} \Delta \mu(\Delta t)^{2}\right\}\left(1-c_{k}\right)
$$

and

$$
\beta=s_{k}(\Delta t)^{2} D_{\mu} \sigma\left\{\Delta \mu V^{2} W^{\prime \prime}+W^{\prime} V\left(1+c_{k}\right)\right\} .
$$

Remark: Note that $\beta$ is the new term we get in the stability polynomial when we go from a uniform to a nonuniform stress in the stability analysis. Observe that $\beta=0$ at $k=k_{\max }$ and therefore (105) becomes

$$
\lambda_{k}^{2}-2\left(1-\Xi_{F L}-\alpha / 2\right) \lambda_{k}+C=0,
$$

which is exactly the same stability polynomial equation we get if we perturb from a uniform- $\sigma-\Delta x$-state. Therefore, the same theorems as before hold.

Theorem 3.1. A sufficient condition for absolute D.-N. instability of (70) at $\left(k_{\max }, N, \Delta t, \Delta \mu, \Delta x, \bar{\sigma}, W^{\prime \prime}, V\right)$, where $V^{2} \Delta \mu(\Delta t)^{2}>0$, is

$$
\bar{\sigma} W^{\prime \prime}<0 \text {. }
$$


Corollary 3.2. Artificial viscosities of the general von Neumann-Richtmyer-Landshoff form, i.e., $q=-\nu a \Delta_{\mu} u$, with $\nu a \geq 0$, cannot make (Yo) at $\left(k_{\max }, N, \Delta t, \Delta \mu, \Delta x, \bar{\sigma}, W^{\prime \prime}, V\right)$ absolutely D.-N. stable when $\overline{p+q} W^{\prime \prime}<0$.

Remark: Computational experiments with typical hypervelocity impacts in solid materials show that the case $\overline{p+q}<0$ and $W^{\prime \prime}>0$ is not unusual and thus Corollary 3.2 . is not vacuous. We see that artificial viscosity cannot overcome the SPH instability. For proofs of Theorem 3.1. and Corollary 3.2. see [8]. 


\subsection{CONSERVATIVE SMOOTHING}

We illustrate the fundamental idea in conservative smoothing using the von Neumann - Richtmyer discrete representation of conservation of volume:

$$
V_{t}=u_{\mu}
$$

where

$$
\left(V_{t}\right)_{j+1 / 2}^{n+1 / 2}=\frac{V_{j+1 / 2}^{n+1}-V_{j+1 / 2}^{n}}{t^{n+1}-t^{n}}
$$

and

$$
\left(u_{\mu}\right)_{j+1 / 2}^{n+1 / 2}=\frac{u_{j+1}^{n+1 / 2}-u_{j}^{n+1 / 2}}{\mu_{j+1}-\mu_{j}} .
$$

For simplicity, consider a uniform material mesh

$$
\mu_{j}=j \Delta \mu, 0 \leq j \leq J
$$

with boundaries

$$
\mu_{\ell b}=\mu_{0}, \mu_{r b}=\mu_{J} .
$$

Remark: Although we here discuss a one-dimensional problem, observe that if we had $J$ mass chunks, $0 \leq j \leq J-1$,

$$
m_{j+1 / 2}=\operatorname{Area}\left(\mu_{j+1}-\mu_{j}\right),
$$

(Area is an area factor whose value we choose as unity for simplicity) with specific volumes, $V_{j+1 / 2}$, distributed throughout a two - or three - dimensional material, then this illustration of the fundamental idea in conservative smoothing would hold, mutatis mutandis, as well in three - or two - as it does in one - dimension(s). Caveat: Observe though, that implicit here is the assumption that a linear ordering has been introduced, (115), along with a tagging of the boundary points, (116). Further, to maintain local smoothing (i.e., to avoid 'action at a distance') suggests that there should be contiguity, in some sense, between $m_{j-1 / 2}$ and $m_{j+1 / 2}$. Furthermore, it seems that $m_{1 / 2}$ and $m_{J-1 / 2}$ should be boundary masses of the two - or three - dimensional problem.

Let's define the midpoint mesh by

$$
\mu_{j+1 / 2}=\left(\mu_{j}+\mu_{j+1}\right) / 2, \text { for } 0 \leq j \leq J-1 .
$$

Now let's do a conservative redefining of the $V_{0}$-distribution on the original mesh to the $\hat{V}_{\text {- }}$-distribution on the on the midpoint mesh by

$$
\hat{V}_{j}=\left(V_{j-1 / 2}+V_{j+1 / 2}\right) / 2, \text { for } 1 \leq j \leq J-1,
$$


with boundary conditions

$$
\hat{V}_{0}=V_{1 / 2}, \quad \hat{V}_{J}=V_{J-1 / 2}
$$

We say that this is a conservative redefining (a.k.a., conservative rezoning) because the total volume before and after is the same, i.e., the sum total volume before is given by

$$
\Sigma_{V \text { before }}=A \sum_{j=0}^{J-1} V_{j+1 / 2}\left(\mu_{j+1}-\mu_{j}\right)
$$

and the sum total volume after is given by

$$
\Sigma_{V a f t e r}=A\left\{\hat{V}_{0}\left(\mu_{1 / 2}-\mu_{0}\right)+\sum_{j=1}^{J-1} \hat{V}_{j}\left(\mu_{j+1 / 2}-\mu_{j-1 / 2}\right)+\hat{V}_{J}\left(\mu_{J}-\mu_{J-1 / 2}\right)\right\}
$$

and it is straightforward to verify that

$$
\Sigma_{V b e f o r e}=\Sigma_{V a f t e r}
$$

Next, we conservatively rezone the $\hat{V}_{\bullet}$-distribution on the midpoint mesh to the $\tilde{V}_{\bullet}$-distribution on the original mesh via

$$
\tilde{V}_{j+1 / 2}=\left(\hat{V}_{j+1}+\hat{V}_{j}\right) / 2, \text { for } 0 \leq j \leq J-1,
$$

which in terms of the original $V_{\bullet}$-distribution is

$$
\tilde{V}_{j+1 / 2}=\left(V_{j+3 / 2}+2 V_{j+1 / 2}+V_{j-1 / 2}\right) / 4, \text { for } 1 \leq j \leq J-2,
$$

with boundary values

$$
\tilde{V}_{1 / 2}=\left(V_{3 / 2}+2 V_{1 / 2}+V_{1 / 2}\right) / 4, \quad \tilde{V}_{J-1 / 2}=\left(V_{J-1 / 2}+2 V_{J-1 / 2}+V_{J-3 / 2}\right) / 4
$$

Note that if we define the 'phantom zone' values $V_{-1 / 2}$ and $V_{J+1 / 2}$ by

$$
V_{-1 / 2}=V_{+1 / 2}, \quad V_{J+1 / 2}=V_{J-1 / 2}
$$

then the single recipe

$$
\tilde{V}_{j+1 / 2}=\left(V_{j+3 / 2}+2 V_{j+1 / 2}+V_{j-1 / 2}\right) / 4, \text { for } 0 \leq j \leq J-1
$$

includes both the interior points, (125), and the boundary points, (126), recipes. Also observe that if we wish to leave the boundary values, $V_{+1 / 2}$ and $V_{J-1 / 2}$, invariant in this form of conservative smoothing, then we may accomplish this with the following recipes. For the interior values of $\tilde{V}_{j+1 / 2}, 2 \leq j \leq J-3$,

$$
\tilde{V}_{j+1 / 2}=\left(V_{j+3 / 2}+2 V_{j+1 / 2}+V_{j-1 / 2}\right) / 4
$$

and for the 'near-boundary' values of $\tilde{V}_{j+1 / 2}, j=1, J-2$,

$$
\tilde{V}_{3 / 2}=\left(V_{5 / 2}+3 V_{3 / 2}\right) / 4, \quad \tilde{V}_{J-3 / 2}=\left(3 V_{J-3 / 2}+V_{J-5 / 2}\right) / 4,
$$


and for the boundary values of $\tilde{V}_{j+1 / 2}, j=0, J-1$

$$
\tilde{V}_{1 / 2}=V_{1 / 2}, \quad \tilde{V}_{J-1 / 2}=V_{J-1 / 2}
$$

Note that we may write (129) as

$$
\tilde{V}=V+c_{s} \Delta_{\mu}^{2} V
$$

where $c_{s}=1 / 4$ and

$$
\left(\Delta_{\mu}^{2} V\right)_{j+1 / 2}=V_{j+3 / 2}-2 V_{j+1 / 2}+V_{j-1 / 2}
$$

Thus the effect of a conservative smoothing of the type here discussed on the von Neumann - Richtmyer representation of the conservation of volume, $V_{t}=u_{\mu}$, would be to transform it to

$$
V_{t}=\left[a c_{s} \Delta \mu / C_{F L}\right] V_{\mu \mu}+u_{\mu}
$$

where

$$
V_{\mu \mu}=\Delta_{\mu}^{2} V /(\Delta \mu)^{2}
$$

We call this conservative smoothing of type d because it introduces a term, $\left[a c_{s} \Delta \mu / C_{F L}\right] V_{\mu \mu}$, which looks like a diffusion term. Consistency with the standard continuum model,

$$
\partial V / \partial t=\partial u / \partial \mu
$$

would require $\left[a c_{s} \Delta \mu / C_{F L}\right] V_{\mu \mu}$ to go to zero as the material and temporal increments vanished.

For further details on conservative smoothing, see [4]. 


\subsection{Stability Analysis with Conservative Smoothing}

Recall that the SPH scheme we are analyzing is

$$
u_{j}^{n+1 / 2}=u_{j}^{n-1 / 2}-\left.\Delta t \frac{D P}{\Delta \mu}\right|_{j} ^{n}, x_{j}^{n+1}=x_{j}^{n}+\Delta t u_{j}^{n+1 / 2}, \quad V_{j}^{n+1}=\left.\frac{D x}{\Delta \mu}\right|_{j} ^{n+1} .
$$

This is equivalent to

$$
\begin{gathered}
u_{j}^{n+1 / 2}=u_{j}^{n-1 / 2}-\left.\Delta t \frac{D P}{\Delta \mu}\right|_{j} ^{n}, x_{j}^{n+1}=x_{j}^{n}+\Delta t u_{j}^{n+1 / 2}, \\
V_{j}^{n+1}=V_{j}^{n}+\left.\Delta t \frac{D u}{\Delta \mu}\right|_{j} ^{n+1 / 2} .
\end{gathered}
$$

We turn off the artificial viscosity and turn on the conservative smoothing of type $d$ with $c_{s}=1 / 4$, i.e.,

$$
\widetilde{\mathbf{U}}_{j}=\mathbf{U}_{j}+\left(\mathbf{U}_{j+1}-2 \mathbf{U}_{j}+\mathbf{U}_{j-1}\right) / 4, \quad \mathbf{U}_{j}=\left(V_{j}, u_{j}, x_{j}\right)^{T} .
$$

Combining (138) and (139) yields the following scheme:

$$
\begin{gathered}
u_{j}^{n+1 / 2}=\tilde{u}_{j}^{n-1 / 2}-\left.\Delta t \frac{D[p(\tilde{V}) A(\widetilde{x})]}{\Delta \mu}\right|_{j} ^{n}, \\
x_{j}^{n+1}=\tilde{x}_{j}^{n}+\Delta t \tilde{u}_{j}^{n+1 / 2}, \quad V_{j}^{n+1}=\tilde{V}_{j}^{n}+\left.\Delta t \frac{D \tilde{u}}{\Delta \mu}\right|_{j} ^{n+1 / 2}
\end{gathered}
$$

The stability polynomial equation becomes

$$
\lambda_{k}^{2}-2 \widetilde{B} \lambda_{k}+\widetilde{C}=0
$$

where

$$
\widetilde{B}=B A_{c s}(k), \quad \widetilde{C}=C A_{c s}^{2}(k)
$$

recall

$$
A_{c s}(k)=\left(1+c_{k}\right) / 2, \quad c_{k}=\cos k \Delta \mu .
$$

Since the artificial viscosity is turned off, we have

$$
C=1, \quad B=1-\Xi_{F L}+i \beta
$$

with

$$
\Xi_{F L}=\left\{\left(1+c_{k}\right)\left[A C_{F L}^{2} / 2+\bar{\sigma} W^{\prime} V(\Delta t)^{2}\right]+\bar{\sigma} W^{\prime \prime} V^{2} \Delta \mu(\Delta t)^{2}\right\}\left(1-c_{k}\right)
$$

and

$$
\beta=s_{k}(\Delta t)^{2} D_{\mu} \sigma\left\{\Delta \mu V^{2} W^{\prime \prime}+W^{\prime} V\left(1+c_{k}\right)\right\}
$$


Lemma 5.0. Given $\lambda^{2}-2 B \lambda+C=0, \lambda_{ \pm}=B \pm\left(B^{2}-C\right)^{1 / 2}, r=\max \left|\lambda_{ \pm}\right|, B=$ $a+i b, a \& b \in \Re, C=0$, then

$$
a^{2}+b^{2} \leq 1 / 4 \Leftrightarrow r \leq 1 .
$$

Proof: Straightforward because $r=2|B|$.

Lemma 5.1. Given $\lambda^{2}-2 B \lambda+C=0, \lambda_{ \pm}=B \pm\left(B^{2}-C\right)^{1 / 2}, r=\max \left|\lambda_{ \pm}\right|, B=$ $a+i b, a \& b \in \Re, b \neq 0, C \geq 0$, then

$$
a^{2}+b^{2}+C / 2 \leq 1 / 4 \Rightarrow r \leq 1 .
$$

Proof: First, the case where $C=0$ is taken care of by Lemma 5.0., so assume henceforth $C>0$. Note that

$$
\lambda_{ \pm}=a+i b \pm\left[(a+i b)^{2}-C\right]^{1 / 2} .
$$

Let $u \& v$ be defined by

$$
\left[(a+i b)^{2}-C\right]^{1 / 2}=u+i v
$$

Therefore,

$$
\left|\lambda_{ \pm}\right|^{2}=(a \pm u)^{2}+(b \pm v)^{2}=a^{2} \pm 2 a u+u^{2}+b^{2} \pm 2 b v+v^{2} .
$$

Square both sides of (163) and compare real and imaginary parts to get

$$
a^{2}-b^{2}-C=u^{2}-v^{2}
$$

and

$$
a b=u v .
$$

If $a \neq 0$, then (153) implies $b / v=u / a$ and thus we see that $\operatorname{sign}(a u)=\operatorname{sign}(b v)$. Since $r^{2}=\max \left|\lambda_{ \pm}\right|^{2}$, it follows that

$$
r^{2}=a^{2}+2|a u|+u^{2}+b^{2}+2|b v|+v^{2}
$$

note that this also holds for $a=0$. Next observe that

$$
2|a u| \leq a^{2}+u^{2}
$$

and therefore

$$
r^{2} \leq 2\left(a^{2}+u^{2}+b^{2}+v^{2}\right)
$$

From (152) and (153):

$$
\left(u^{2}+v^{2}\right)^{2}=\left(a^{2}-b^{2}-C\right)^{2}+4 a^{2} b^{2}=\left(a^{2}+b^{2}+C\right)^{2}-4 C a^{2} .
$$


Then using the fact that $C>0$ yields

$$
u^{2}+v^{2} \leq a^{2}+b^{2}+C
$$

and thus

$$
r^{2} \leq 4\left(a^{2}+b^{2}+C / 2\right)
$$

The remainder of the proof is straightforward.

Lemma 5.2. Given $\lambda^{2}-2 B \lambda+C=0, \lambda_{ \pm}=B \pm\left(B^{2}-C\right)^{1 / 2}, r=\max \left|\lambda_{ \pm}\right|, B=$ $a+i b, a \& b \& C \in \Re, a \neq 0 \neq b$, then

$$
r \leq 1 \Leftrightarrow|B|^{2}-C+\left[\left(|B|^{2}-C\right)^{2}+4 b^{2} C\right]^{1 / 2} \leq(1-C)^{2} / 2 .
$$

Proof: Express the roots in polar form, let

$$
\lambda_{1}=\rho e^{i \theta}, \lambda_{2}=\frac{C}{\rho} e^{-i \theta}
$$

and, without loss of generality. take $\rho \geq \frac{C}{\rho}$, and therefore $\rho=r$. Note that

$$
\rho e^{i \theta}+\frac{C}{\rho} e^{-i \theta}=2(a+i b) .
$$

Compare real, i.e.,

$$
\left(\rho+\frac{C}{\rho}\right) \cos \theta=2 a
$$

and imaginary parts, i.e.,

$$
\left(\rho-\frac{C}{\rho}\right) \sin \theta=2 b .
$$

Observe that $a \neq 0 \Rightarrow \cos \theta \neq 0$ and $b \neq 0 \Rightarrow \sin \theta \neq 0$ and $\rho>\frac{C}{\rho}$. Therefore,

$$
\rho+\frac{C}{\rho}=2 a / \cos \theta>0
$$

and

$$
\rho-\frac{C}{\rho}=2 b / \sin \theta>0
$$

Adding the prior pair of equations yields

$$
\rho=a / \cos \theta+b / \sin \theta .
$$


Multiply equations (152) and (153) by $\rho$ to get

$$
\rho^{2}-2 a \rho / \cos \theta+C=0
$$

and

$$
\rho^{2}-2 b \rho / \sin \theta-C=0 .
$$

Writing down the roots, since $\rho>\frac{C}{\rho}$, we have

$$
\rho=a / \cos \theta+\left[(a / \cos \theta)^{2}-C\right]^{1 / 2}, \quad \frac{C}{\rho}=a / \cos \theta-\left[(a / \cos \theta)^{2}-C\right]^{1 / 2},
$$

and

$$
\rho=b / \sin \theta+\left[(b / \sin \theta)^{2}+C\right]^{1 / 2}, \quad \frac{C}{\rho}=b / \sin \theta-\left[(b / \sin \theta)^{2}+C\right]^{1 / 2} .
$$

From (154) and (158) we get

$$
a / \cos \theta+b / \sin \theta=a / \cos \theta+\left[(a / \cos \theta)^{2}-C\right]^{1 / 2}
$$

and therefore

$$
(b / \sin \theta)^{2}=(a / \cos \theta)^{2}-C
$$

Let $y \equiv(\sin \theta)^{2}$ to get

$$
C y^{2}+\left(a^{2}+b^{2}-C\right) y-b^{2}=0 .
$$

Note that if $C=0$, then $y=b^{2} /\left(a^{2}+b^{2}\right)$ and this picks the correct root of the quadratic, i.e.,

$$
(\sin \theta)^{2}=\frac{2 b^{2}}{a^{2}+b^{2}-C+\left[\left(a^{2}+b^{2}-C\right)^{2}+4 b^{2} C\right]^{1 / 2}}
$$

From (159)

$$
\rho \leq 1 \Leftrightarrow b / \sin \theta+\left[(b / \sin \theta)^{2}+C\right]^{1 / 2} \leq 1
$$

and therefore

$$
\rho \leq 1 \Leftrightarrow(b / \sin \theta)^{2}+C \leq(1-b / \sin \theta)^{2} \Leftrightarrow 2 b / \sin \theta \leq 1-C
$$

and thus

$$
\rho \leq 1 \Leftrightarrow 4 b^{2} \leq(1-C)^{2}(\sin \theta)^{2}
$$

Now put (164) with this to get

$$
\rho \leq 1 \Leftrightarrow 2\left\{a^{2}+b^{2}-C+\left[\left(a^{2}+b^{2}-C\right)^{2}+4 b^{2} C\right]^{1 / 2}\right\} \leq(1-C)^{2} .
$$


The remainder of the proof is straightforward.

Remark: Before stating the next theorem, some explanation of the theorem's form appears to be appropriate. To allow for the proper modelling of physical instabilities, it seems reasonable to allow bounded growth for the amplitudes of the Fourier components of the lesser wave numbers, i.e., $|k|<\theta k_{\max }$, where $0<\theta<1$, and only require absolute D. - N. stability for the larger wave numbers, i.e., $\theta k_{\max } \leq|k| \leq k_{\max }$. If $\beta=0$ in (146), then the stability polynomial is the same as if we perturb from a uniform- $\sigma$ - $\Delta x$-state and thus we have the following proposition from that prior analysis.

Theorem 5.1. When $\beta=0$ in (146), a sufficient condition for absolute D.-N. stability of (140) at $\left(k, \Delta t, \Delta \mu, N, \mathbf{C}^{u}\right)$ for $\frac{1}{2} k_{\max } \leq|k| \leq k_{\max }$ is as follows:

$$
2\left|\xi_{F L}\right|+4\left|W_{s t u f f}^{\prime \prime}\right| \leq 1,
$$

with

$$
\xi_{F L}=-2\left(a^{2}-\rho \bar{\sigma}\right)(\Delta t / \Delta \mu)^{2} W^{\prime} V^{2} \Delta \mu^{2}
$$

and

$$
W_{s t u f f}^{\prime \prime}=\bar{\sigma} W^{\prime \prime} V^{2} \Delta t^{2} \Delta \mu .
$$

Proof: See [8].

Theorem 5.2. When $\beta \neq 0$ in (146), a sufficient condition for absolute D.-N. stability of (140) at $\left(k, \Delta t, \Delta \mu, N, \mathrm{C}^{u}\right)$ for $\frac{2}{3} k_{\max } \leq|k| \leq k_{\max }$ is as follows:

$$
\left|\xi_{F L}\right|+4\left|W_{s t u f f}^{\prime \prime}\right| \leq \sqrt{7}-2
$$

and

$$
\left|\eta_{D \sigma}\right|+4\left|W_{\text {new }}^{\prime \prime}\right| \leq 4 \sqrt{7 / 3}
$$

where

$$
\xi_{F L}=-2\left(a^{2}-\rho \bar{\sigma}\right)(\Delta t / \Delta \mu)^{2} W^{\prime} V^{2} \Delta \mu^{2}
$$

and also

$$
W_{s t u f f}^{\prime \prime}=\bar{\sigma} W^{\prime \prime} V^{2} \Delta t^{2} \Delta \mu
$$

further

$$
\eta_{D \sigma}=2 W^{\prime} V \Delta t^{2} D_{\mu} \sigma
$$

and finally

$$
W_{\text {new }}^{\prime \prime}=W^{\prime \prime} V^{2} \Delta t^{2} \Delta \mu D_{\mu} \sigma .
$$


Proof: Note that $\frac{2}{3} k_{\max } \leq|k| \leq k_{\max }$ implies

$$
0 \leq A_{c s}(k) \leq 1 / 4 \text {. }
$$

Since $\beta \neq 0$, the stability polynomial can only have distinct roots, therefore the sufficient condition for absolute D.-N. stability is $r \leq 1$. Recall that the stability polynomial equation is

$$
\lambda_{k}^{2}-2 \tilde{B} \lambda_{k}+\widetilde{C}=0
$$

where

$$
\widetilde{B}=\left(1-\Xi_{F L}+i \beta\right) A_{c s}, \quad \tilde{C}=A_{c s}^{2},
$$

with

$$
\begin{gathered}
\Xi_{F L}=\left(1-c_{k}\right)\left[\xi_{F L} A_{c s}+W_{s t u f f}^{\prime \prime}\right], \quad A=-2 W^{\prime} V^{2} \Delta \mu^{2}, \\
\xi_{F L}=A C_{F L}^{2}+2 \bar{\sigma} W^{\prime} V \Delta t^{2}, W_{s t u f f}^{\prime \prime}=\bar{\sigma} W^{\prime \prime} V^{2} \Delta \mu \Delta t^{2}
\end{gathered}
$$

and

$$
\text { (193) } \beta=s_{k}\left(\eta_{D \sigma} A_{c s}+W_{n e w}^{\prime \prime}\right), \quad \eta_{D \sigma}=2 \Delta t^{2} D_{\mu} \sigma W^{\prime} V, \quad W_{n e w}^{\prime \prime}=\Delta t^{2} D_{\mu} \sigma \Delta \mu V^{2} W^{\prime \prime}
$$

Observe that

$$
|\tilde{B}|^{2}=\left[\left(1-\Xi_{F L}\right)^{2}+\beta^{2}\right] A_{c s}^{2}
$$

If

$$
\left(1-\Xi_{F L}\right)^{2} \leq 7 / 4
$$

and

$$
\beta^{2} \leq 7 / 4
$$

then

$$
|\tilde{B}|^{2}+\tilde{C} / 2 \leq 1 / 4
$$

which, by Lemma 5.1, is a sufficient condition for absolute D.-N. stability. Thus we see that (195) and (196) are sufficient for stability. Now we seek sufficient conditions for (195) and (196). Note that

$$
\left(1-\Xi_{F L}\right)^{2} \leq 7 / 4 \Leftrightarrow 1-\sqrt{7} / 2 \leq \Xi_{F L} \leq 1+\sqrt{7} / 2
$$

and then, by the definition of $\Xi_{F L},(198)$ is equivalent to

$$
(2-\sqrt{7}) / 2 \leq\left(1-c_{k}\right)\left[\xi_{F L} A_{c s}+W_{s t u f f}^{\prime \prime}\right] \leq(2+\sqrt{7}) / 2 .
$$


If $\frac{2}{3} k_{\max } \leq|k| \leq k_{\max }$, then

$$
3 / 2 \leq 1-c_{k} \leq 2
$$

and therefore we have

$$
(2-\sqrt{7}) / 4 \leq \xi_{F L} A_{c s}+W_{s t u f f}^{\prime \prime} \leq(2+\sqrt{7}) / 4 .
$$

First, consider the righthand inequality in (201), recall that $A_{c s} \in[0,1 / 4]$, and note that there are two cases, i.e.,

$$
\text { Case(+): } \xi_{F L}>0 \text { and Case(-): } \xi_{F L} \leq 0 \text {. }
$$

In $\operatorname{Case}(+): \xi_{F L}>0:$

$$
\xi_{F L} / 4+W_{s t u f f}^{\prime \prime} \leq(2+\sqrt{7}) / 4 \Rightarrow \xi_{F L} A_{c s}+W_{s t u f f}^{\prime \prime} \leq(2+\sqrt{7}) / 4 .
$$

In $C a s e(-): \xi_{F L} \leq 0$ :

$$
W_{s t u f f}^{\prime \prime} \leq(2+\sqrt{7}) / 4 \Rightarrow \xi_{F L} A_{c s}+W_{s t u f f}^{\prime \prime} \leq(2+\sqrt{7}) / 4 .
$$

Using the fact that $\max (a, b)=(a+b) / 2+|a-b| / 2$, we get

$$
\xi_{F L} / 8+W_{s t u f f}^{\prime \prime}+\left|\xi_{F L}\right| / 8 \leq(2+\sqrt{7}) / 4 \Rightarrow \xi_{F L} A_{c s}+W_{s t u f f}^{\prime \prime} \leq(2+\sqrt{7}) / 4
$$

for all $A_{c s} \in[0,1 / 4]$.

Second, consider the lefthand inequality in (201) and again consider the two cases. In $\operatorname{Case}(+): \xi_{F L}>0$ :

$$
(2-\sqrt{7}) / 4 \leq W_{s t u f f}^{\prime \prime} \Rightarrow(2-\sqrt{7}) / 4 \leq \xi_{F L} A_{c s}+W_{s t u f f}^{\prime \prime} .
$$

In Case(-): $\xi_{F L} \leq 0$ :

$$
(2-\sqrt{7}) / 4 \leq \xi_{F L} / 4+W_{s t u f f}^{\prime \prime} \Rightarrow(2-\sqrt{7}) / 4 \leq \xi_{F L} A_{c s}+W_{s t u f f}^{\prime \prime} .
$$

Using the fact that $\min (a, b)=(a+b) / 2-|a-b| / 2$, we get

$$
(2-\sqrt{7}) / 4 \leq \xi_{F L} / 8+W_{s t u f f}^{\prime \prime}-\left|\xi_{F L}\right| / 8 \Rightarrow(2-\sqrt{7}) / 4 \leq \xi_{F L} A_{c s}+W_{s t u f f}^{\prime \prime}
$$

for all $A_{c s} \in[0,1 / 4]$.

Next note that

$$
\begin{gathered}
(2-\sqrt{7}) / 4 \leq \xi_{F L} / 8+W_{s t u f f}^{\prime \prime}-\left|\xi_{F L}\right| / 8 \\
\Leftrightarrow \\
\xi_{F L} / 8-W_{s t u f f}^{\prime \prime}-\left|\xi_{F L}\right| / 8 \leq(-2+\sqrt{7}) / 4 .
\end{gathered}
$$

Thus both inequalities in (201) are satisfied provided

$$
\xi_{F L} / 8-W_{s t u f f}^{\prime \prime}-\left|\xi_{F L}\right| / 8 \leq(-2+\sqrt{7}) / 4
$$


and

$$
\xi_{F L} / 8+W_{s t u f f}^{\prime \prime}+\left|\xi_{F L}\right| / 8 \leq(2+\sqrt{7}) / 4 .
$$

Observe that (183) suffices for both (209) and (210).

Next recall that we need a sufficient condition for

$$
\beta^{2} \leq 7 / 4
$$

and this leads us to consider

$$
-\sqrt{7} / 2 \leq \beta \leq \sqrt{7} / 2
$$

Recall that

$$
\beta=s_{k}\left(\eta_{D \sigma} A_{c s}+W_{n e w}^{\prime \prime}\right)
$$

and therefore we want a sufficient condition for

$$
-\sqrt{7} / 2 \leq s_{k}\left(\eta_{D \sigma} A_{c s}+W_{n e w}^{\prime \prime}\right) \leq \sqrt{7} / 2
$$

for $\frac{2}{3} k_{\max } \leq|k| \leq k_{\max }$ where

$$
0 \leq s_{k} \leq \sqrt{3} / 2
$$

Thus we see that we want a sufficient condition for

$$
-\sqrt{7 / 3} \leq \eta_{D \sigma} A_{c s}+W_{n e w}^{\prime \prime} \leq \sqrt{7 / 3}
$$

As before, we first consider the righthand inequality in (216), recall that $A_{c s} \in$ $[0,1 / 4]$, and the two cases are

$$
\text { Case(+): } \eta_{D \sigma}>0 \text { and Case(-): } \eta_{D \sigma} \leq 0 \text {. }
$$

In $C a s e(+): \eta_{D \sigma}>0$ :

$$
\eta_{D \sigma} / 4+W_{\text {new }}^{\prime \prime} \leq \sqrt{7 / 3} \Rightarrow \eta_{D \sigma} A_{c s}+W_{n e w}^{\prime \prime} \leq \sqrt{7 / 3}
$$

In $\operatorname{Case}(-): \eta_{D_{\sigma}} \leq 0$

$$
W_{n e w}^{\prime \prime} \leq \sqrt{7 / 3} \Rightarrow \eta_{D \sigma} A_{c s}+W_{n e w}^{\prime \prime} \leq \sqrt{7 / 3}
$$

Again as before, we use $\max (a, b)=(a+b) / 2+|a-b| / 2$ to get

$$
\eta_{D \sigma} / 8+W_{\text {new }}^{\prime \prime}+\left|\eta_{D \sigma}\right| / 8 \leq \sqrt{7 / 3} \Rightarrow \eta_{D \sigma} A_{c s}+W_{n e w}^{\prime \prime} \leq \sqrt{7 / 3}
$$

Second, proceeding in parallel to the previous analysis, we consider the lefthand inequality in (216). In $\operatorname{Case}(+): \eta_{D \sigma}>0$ :

$$
-\sqrt{7 / 3} \leq W_{\text {new }}^{\prime \prime} \Rightarrow \underset{26}{-\sqrt{7 / 3}} \leq \eta_{D \sigma} A_{c s}+W_{\text {new }}^{\prime \prime} .
$$


In $\operatorname{Case}(-): \eta_{D \sigma} \leq 0$

$$
-\sqrt{7 / 3} \leq \eta_{D \sigma} / 4+W_{n e w}^{\prime \prime} \Rightarrow-\sqrt{7 / 3} \leq \eta_{D \sigma} A_{c s}+W_{n e w}^{\prime \prime}
$$

Yet again as before, using $\min (a, b)=(a+b) / 2-|a-b| / 2$, we get

$$
-\sqrt{7 / 3} \leq \eta_{D \sigma} / 8+W_{n e w}^{\prime \prime}-\left|\eta_{D \sigma}\right| / 8 \Rightarrow-\sqrt{7 / 3} \leq \eta_{D \sigma} A_{c s}+W_{n e w}^{\prime \prime}
$$

for all $A_{c s} \in[0,1 / 4]$. Note now that

$$
\eta_{D \sigma} / 8-W_{n e w}^{\prime \prime}-\left|\eta_{D \sigma}\right| / 8 \leq \sqrt{7 / 3}
$$

and

$$
\eta_{D \sigma} / 8+W_{n e w}^{\prime \prime}+\left|\eta_{D \sigma}\right| / 8 \leq \sqrt{7 / 3}
$$

suffice for both inequalities in (216) and, in turn,

$$
\left|\eta_{D \sigma}\right|+4\left|W_{n e w}^{\prime \prime}\right| \leq 4 \sqrt{7 / 3}
$$

suffices for both (223) and(224). Q.E.D.

We now apply Lemma 5.2 to obtain a necessary and sufficient condition for stability when $\beta \neq 0$.

Theorem 5.3. When $\beta \neq 0$ in (146), a necessary and sufficient condition for absolute D.-N. stability of (140) at $\left(k, \Delta t, \Delta \mu, N, \mathrm{C}^{u}\right)$ for $0 \leq|k| \leq k_{\max }$ is as follows:

$$
|\widetilde{B}|^{2}-A_{c s}^{2}+\left[\left(|\widetilde{B}|^{2}-A_{c s}^{2}\right)^{2}+4 \beta^{2} A_{c s}^{4}\right]^{1 / 2} \leq\left(1-A_{c s}^{2}\right)^{2} / 2
$$

where

$$
|\tilde{B}|^{2}=\left\{\left(1-\Xi_{F L}\right)^{2}+\beta^{2}\right\} A_{c s}^{2}, \quad A_{c s}=\left(1+c_{k}\right) / 2,
$$

with

$$
\begin{gathered}
\Xi_{F L}=\left(1-c_{k}\right)\left[\xi_{F L} A_{c s}+W_{s t u f f}^{\prime \prime}\right], \quad \xi_{F L}=A C_{F L}^{2}+2 \bar{\sigma} W^{\prime} V \Delta t^{2}, \\
A=-2 W^{\prime} V^{2} \Delta \mu^{2}, \quad W_{s t u f f}^{\prime \prime}=\bar{\sigma} W^{\prime \prime} V^{2} \Delta \mu \Delta t^{2}
\end{gathered}
$$

further

$(229) \beta=s_{k}\left(\eta_{D \sigma} A_{c s}+W_{n e w}^{\prime \prime}\right), \quad \eta_{D \sigma}=2 \Delta t^{2} D_{\mu} \sigma W^{\prime} V, \quad W_{n e w}^{\prime \prime}=\Delta t^{2} D_{\mu} \sigma \Delta \mu V^{2} W^{\prime \prime}$

and finally

$$
c_{k}=\cos \pi k / k_{\max }, \quad s_{k}=\sin \pi k / k_{\max } .
$$


Proof: Recall that Lemma 5.2. says: Given $\lambda^{2}-2 B \lambda+C=0, \lambda_{ \pm}=B \pm\left(B^{2}-\right.$ $C)^{1 / 2}, r=\max \left|\lambda_{ \pm}\right|, B=a+i b, a \& b \& C \in \Re, a \neq 0 \neq b$, then

$$
r \leq 1 \Leftrightarrow|B|^{2}-C+\left[\left(|B|^{2}-C\right)^{2}+4 b^{2} C\right]^{1 / 2} \leq(1-C)^{2} / 2 .
$$

Then substitute $\widetilde{B}$ for $B$, where $\widetilde{B}=\tilde{a}+i \tilde{b}$ and

$$
\tilde{a}=\left(1-\Xi_{F l}\right) A_{c s}, \quad \tilde{b}=\beta A_{c s}, \quad C=A_{c s}^{2}
$$

to get (226). Further, recall that the stability polynomial can only have distinct roots for $\beta \neq 0$; therefore, $r \leq 1$ is necessary and sufficient. Q.E.D.

Corollary 5.3A. When $\beta \neq 0$ in (146), a necessary and sufficient condition for absolute D.-N. stability of $(140)$ at $\left(k, \Delta t, \Delta \mu, N, \mathbf{C}^{u}\right)$ for $0 \leq|k| \leq k_{\max }$ is as follows:

$$
A_{c s}^{2}\left[\left(1-|B|^{2}\right)^{2}+4 \beta^{2}\right]^{1 / 2} \leq A_{c s}^{2}\left(1-|B|^{2}\right)+\left(1-A_{c s}^{2}\right)^{2} / 2,
$$

where

$$
|B|^{2}=\left\{\left(1-\Xi_{F L}\right)^{2}+\beta^{2}\right\} .
$$

Proof: Straightforward from Theorem 5.3.

Corollary 5.3B. When $\beta \neq 0$ in (146), a necessary and sufficient condition for absolute D.-N. stability of $(140)$ at $\left(k, \Delta t, \Delta \mu, N, \mathrm{C}^{u}\right)$ for $0 \leq|k| \leq k_{\max }$ is as follows:

$$
\left(-2 \Xi_{F L}+\Xi_{F L}^{2}\right) A_{c s}^{2}\left(1-A_{c s}^{2}\right)^{2}+\beta^{2}\left[A_{c s}^{2}\left(1-A_{c s}^{2}\right)^{2}+4 A_{c s}^{4}\right] \leq\left(1-A_{c s}^{2}\right)^{4} / 4
$$

Proof: Square both sides of (233), cancel like terms, and expand $|B|^{2}$.

Corollary 5.3C. When $\beta \neq 0$ in (146), a sufficient condition for absolute D.-N. stability of (140) at $\left(k, \Delta t, \Delta \mu, N, \mathbf{C}^{u}\right)$ for $k_{\max } / 2 \leq|k| \leq k_{\max }$ is as follows:

$$
\begin{gathered}
\left\{|| \xi_{F L}|+2| W_{s t u f f}^{\prime \prime}||+\left(\left|\xi_{F L}\right| / 2+\left|W_{s t u f f}^{\prime \prime}\right|\right)^{2}\right\}\left(\frac{3}{4}\right)^{2} \\
+\left(\left|\eta_{D \sigma}\right| / 2+\left|W_{\text {new }}^{\prime \prime}\right|\right)^{2}\left[\left(\frac{3}{4}\right)^{2}+1\right] \leq\left(\frac{3}{4}\right)^{4}
\end{gathered}
$$

Proof: $k_{\max } / 2 \leq|k| \leq k_{\max } \Rightarrow 0 \leq A_{c s}(k) \leq 1 / 2 \& 0 \leq s_{k} \leq 1 \& 0 \leq c_{k} \leq 1$. The rest is straightforward from Corollary $5.3 \mathrm{~B}$. 


\subsection{Results of Computational Experiments}

The test problem here involves the collision of two mirror-symmetric shock waves; it is a special case of the Riemann problem and is also a variation on a test problem introduced in [5]. Proceeding from left to right, the initial values are the lefthand state,

$$
\begin{gathered}
p_{\ell}=1.35 \times 10^{4} \text { dynes } / \mathrm{cm}^{2}, \quad V_{\ell}=7.5 \times 10^{5} \mathrm{~cm}^{3} / \mathrm{gm}, \\
u_{\ell}=2.958 \times 10^{4} \mathrm{~cm} / \mathrm{sec}
\end{gathered}
$$

connected by a rightward shock to the initial middle state,

$$
p_{0}=10^{4} \text { dynes } / \mathrm{cm}^{2}, \quad V_{0}=10^{6} \mathrm{~cm}^{3} / \mathrm{gm}, u_{0}=0,
$$

which in turn is connected by a leftward shock to the righthand state,

$$
\begin{gathered}
p_{r}=1.35 \times 10^{4} \text { dynes } / \mathrm{cm}^{2}, \quad V_{r}=7.5 \times 10^{5} \mathrm{~cm}^{3} / \mathrm{gm}, \\
u_{r}=-2.958 \times 10^{4} \mathrm{~cm} / \mathrm{sec} .
\end{gathered}
$$

After collision, the lefthand state is connected by a leftward shock to the final middle state,

$$
p_{m}=1.7 \times 10^{4} \text { dynes } / \mathrm{cm}^{2}, \quad V_{m}=5 \times 10^{5} \mathrm{~cm}^{3} / \mathrm{gm}, \quad u_{m}=0,
$$

which in turn is connected by a rightward shock to the righthand state. In this test problem we have a uniform material increment, i.e.,

$$
\Delta \mu=10^{-4} \mathrm{gm} / \mathrm{cm}^{2} .
$$

The material law is Hooke's law. The CFL number is

$$
C_{F L}=.2
$$

The program (see [14] for a program listing) computes the exact solution, vNR solution, and S.P.H. solution at collision and after collision and compares the nondimensional, normalized $\ell_{1}$-norm of the difference between the exact solution and the vNR solution; it also computes this error measure for the S.P.H. solution.

ERROR MEASURES: Let $U_{\text {excon }}$ be the exact continuum solution and $U_{\text {dcomp }}$ be the digital computer solution, where $U$ may be specific volume, specific momentum, specific energy, et cetera. Let $U_{\max }$ be the maximum magnitude of $U_{\text {excon }}$ and

$$
\Delta_{e r r} U \equiv \sum_{j=1}^{n z o n e s}\left|U_{e x c o n}(j)-U_{d c o m p}(j)\right| /\left(U_{\max } * n z o n e s\right) .
$$


For these test problems

$$
\text { nzones }=200 \text {. }
$$

ERROR COMPARISONS: The $\Delta_{e r r} U$ numbers in the following table are for: the $v N R_{0,0}$ code (a von Neumann-Richtmyer code using artificial viscosity coefficients $c_{1}=0, c_{2}=0$ ); the $v N R_{0,02}$ code (using $c_{1}=0, c_{2}=.02$ ); the SPHCS hydrocode is the S.P.H. code with conservative smoothing as follows. First, full $\left(c_{s}=1 / 4\right)$ conservative smoothing of type $\mathrm{d}$ was done three times on the initial data. Thereafter, the conservative smoothing coefficient at $\mu_{j+1 / 2}$ was given by

$$
c_{s j+1 / 2}=\min \left\{1 / 4,5 \times 10^{-4} \max \left[1, \frac{h^{2}}{10^{-12}+\left(x_{j+1}-x_{j}\right)^{2}}\right]\right\},
$$

where $h$ is the smoothing length and, for these calculations,

$$
h=100 \mathrm{~cm} \text {. }
$$

By the way, these calculations used the B-Spline Kernel, i.e.,

$$
W^{\prime}(x)=\frac{1}{h^{2}}\left\{\begin{array}{ll}
2\left[-\frac{x}{h}+\frac{3}{4}\left(\frac{x}{h}\right)^{2}\right], & \text { if } 0 \leq x \leq h \\
-\frac{1}{2}\left[2-\frac{x}{h}\right]^{2}, & \text { if } h \leq x \leq 2 h \\
0, & \text { otherwise }
\end{array}\right\}
$$

With respect to the conservative smoothing: the smoothing recipe is

$$
U_{j}=U_{j}+c_{s j+1 / 2}\left(U_{j+1}-U_{j}\right)-c_{s j-1 / 2}\left(U_{j-1}-U_{j}\right)
$$

with $c_{s j+1 / 2}$ as defined in (245). Further details and a listing of the $v N R_{0,0}, v N R_{0,02}$ and $S P H C S$ hydrocodes may be found in [14].

Comparison of $\Delta_{e r r} U$ Values At Collision:

$t_{c o l}=2.789 \times 10^{-2}$ seconds; $n=1680$ cycles :

$\begin{array}{ccccc}\text { Quantity } & v N R_{0,0} & v N R_{0,02} & \text { SPHCS } & \text { worst/best } \\ u & 1.585 \times 10^{-4} & 1.588 \times 10^{-4} & 1.284 \times 10^{-4} & 1.24 \\ V & 4.834 \times 10^{-5} & 4.840 \times 10^{-5} & 2.616 \times 10^{-5} & 1.85 \\ p & 3.759 \times 10^{-5} & 3.764 \times 10^{-5} & 2.035 \times 10^{-5} & 1.85\end{array}$

Observe that in all cases $S P H C S$, i.e., the S.P.H. code with conservative smoothing, gives the least error. The last column in the table above is the ratio of the worst (i.e., the largest) to the best (i.e., the least) error; this provides a quantitative measure of how much improvement conservative smoothing can make. For example, in the $V$ row we have the worst error occurring in the $v N R_{0,02}$ code with $\Delta_{\text {err }} V \doteq 4.840 \times 10^{-5}$ and the best error occurring in the $S P H C S$ code with $\Delta_{\text {err }} V \doteq 2.616 \times 10^{-5}$; the 
ratio of worst to best here is approximately 1.85 . Thus, by this measure, the $S P H C S$ code, an S.P.H. code with conservative smoothing, did almost two times better, with respect to $V$-accuracy, than the $v N R_{0,02}$ code, a von Neumann-Richtmyer code with artificial viscosity coefficients $c_{1}=0, c_{2}=.02, C_{F L}=.2$ and on a Hooke's law test problem. Recall that the von Neumann-Richtmyer-Landshoff (see Landshoff (1955)) artificial viscosity is of the form

$$
q=-c_{1} a \Delta_{\mu} u-c_{2} \rho\left|\Delta_{\mu} u\right| \Delta_{\mu} u
$$

Our computational experiments indicate that, on this test problem (with Hooke's law and $C_{F L}=.2$ ) the vNR code gets smaller errors with the artificial viscosity turned off. We also know that the vNR codes get smaller errors as $C_{F L} \rightarrow 1$ but we wanted to compare the SPH code with the vNR code at the same $C_{F L}$ number, hence these comparisons. Someone might also protest that this is a rather 'wimpy' test problem and we confess that we agree; we intend eventually to do more extensive testing and include problems with stronger shocks and more difficult material laws such as ideal gas, Mie-Grüneisen, et cetera.

\begin{tabular}{ccccc}
\multicolumn{6}{c}{ Comparison of $\Delta_{\text {err }} U$ Values After Collision : } \\
$t_{\text {aftcol }}=4.225 \times 10^{-2}$ seconds; $n=2530$ cycles : \\
Quantity & $v N R_{0,0}$ & $v N R_{0,02}$ & $S P H C S$ & worst $/$ best \\
$u$ & $2.296 \times 10^{-4}$ & $2.300 \times 10^{-4}$ & $1.738 \times 10^{-4}$ & 1.32 \\
$V$ & $6.960 \times 10^{-5}$ & $6.975 \times 10^{-5}$ & $5.692 \times 10^{-5}$ & 1.23 \\
$p$ & $4.889 \times 10^{-5}$ & $4.899 \times 10^{-5}$ & $4.002 \times 10^{-5}$ & 1.22
\end{tabular}

From the 'worst/best' column, we see that the SPHCS code is doing from about $22 \%$ to about $32 \%$ better than the $v N R_{0,02}$ code by these error measures on this test problem.

GRAPHICAL PRESENTATIONS: Now we present the results in graphical form. 
The next table provides a 'table of contents' for the graphs which follow.

$\begin{array}{cc}\text { FIGURE } & \text { DESCRIPTION } \\ 6.1 \text { top left } & u_{\text {excon }} \text { at } t=0 \\ 6.1 \text { top right } & V_{\text {excon }} \text { at } t=0 \\ 6.1 \text { bottom } & p_{\text {excon at } t=0} \\ 6.2 & v N R \text { error versus } c_{2} \\ 6.3 & v N R_{0,0} u \text { at } t=t_{c o l} \\ 6.4 & S P H C S u \text { at } t=t_{c o l} \\ 6.5 & v N R_{0,0} V \text { at } t=t_{c o l} \\ 6.6 & S P H C S V \text { at } t=t_{c o l} \\ 6.7 & v N R_{0,0} p \text { at } t=t_{c o l} \\ 6.8 & S P H C S p \text { at } t=t_{c o l} \\ 6.9 & v N R_{0,0} u \text { at } t=t_{\text {aftcol }} \\ 6.10 & S P H C S u \text { at } t=t_{a f t c o l} \\ 6.11 & v N R_{0,0} V \text { at } t=t_{a f t c o l} \\ 6.12 & S P H C S V \text { at } t=t_{a f t c o l} \\ 6.13 & v N R_{0,0} p \text { at } t=t_{a f t c o l} \\ 6.14 & S P H C S p \text { at } t=t_{a f t c o l}\end{array}$



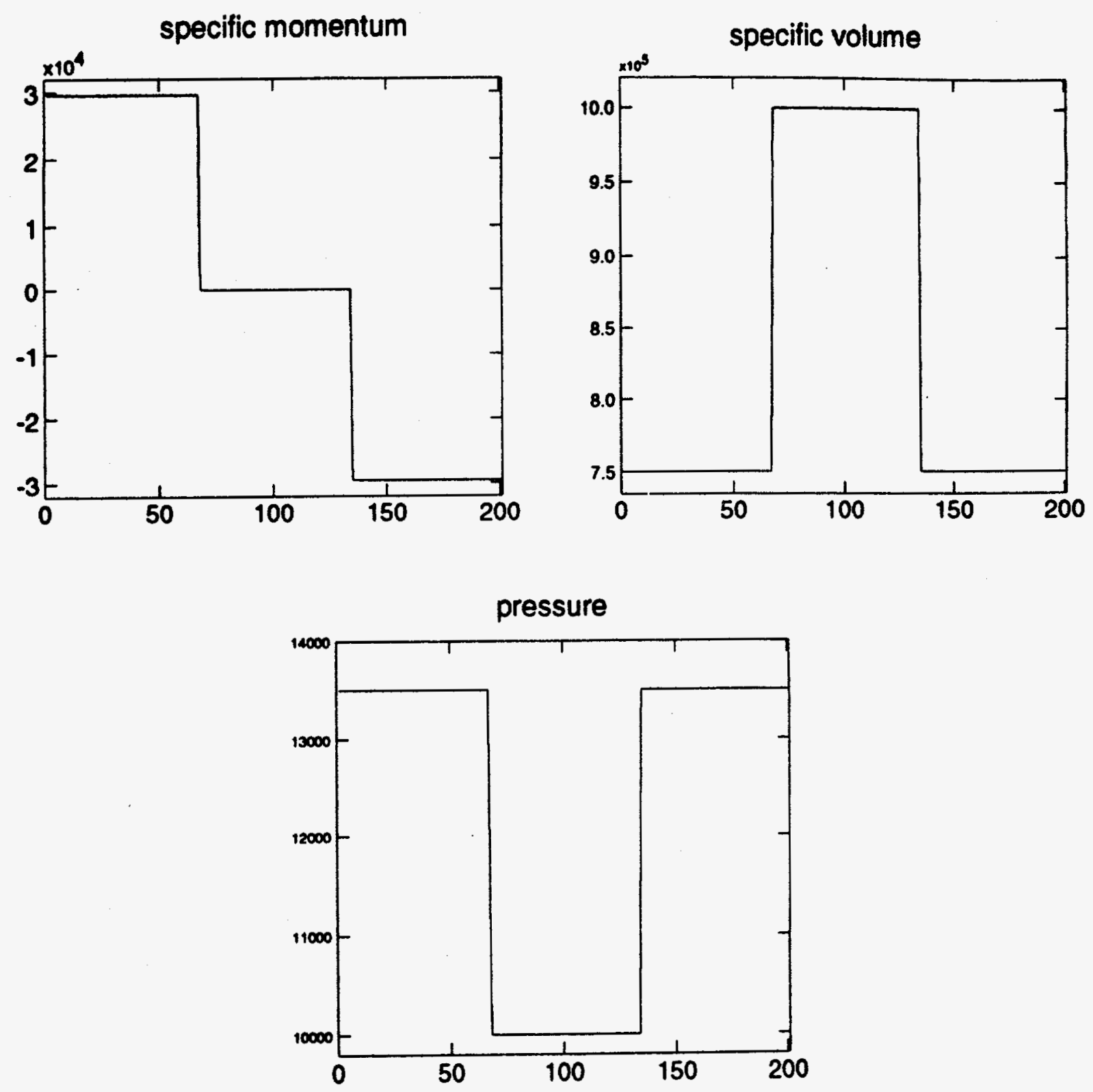

INITIAL DATA OF TEST PROBLEM

Figure 6.1 


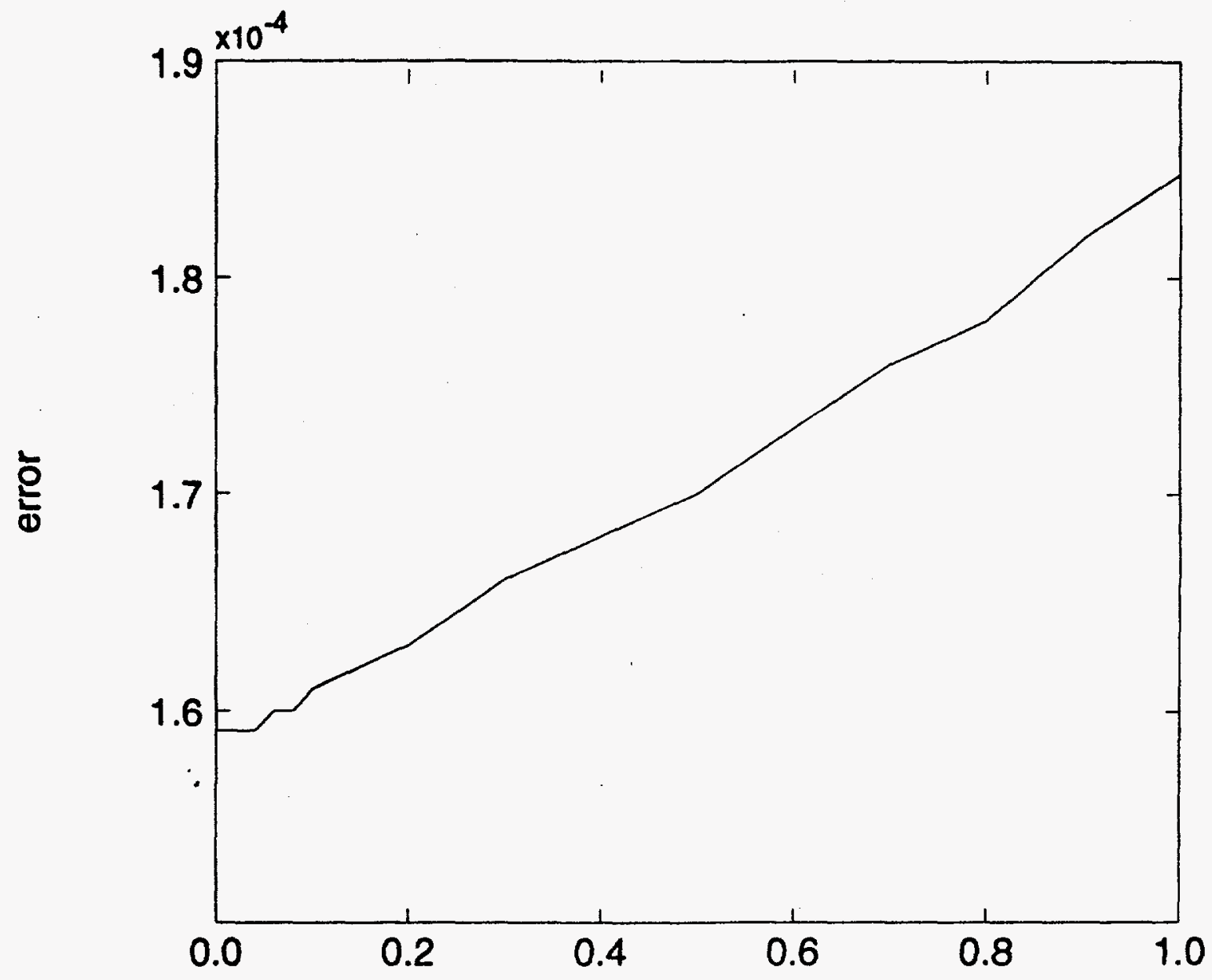

C2

$x$-coordinate: $C 2$ in artificial viscosity

$y$-coordinate: $1-1$ norm of error of specific momentum at collision

Conclusion: vNR gives us better results with artificial viscosity turned off when we use Hooke's Law as our material law and with $C_{F L}=0.2$.

Figure 6.2 


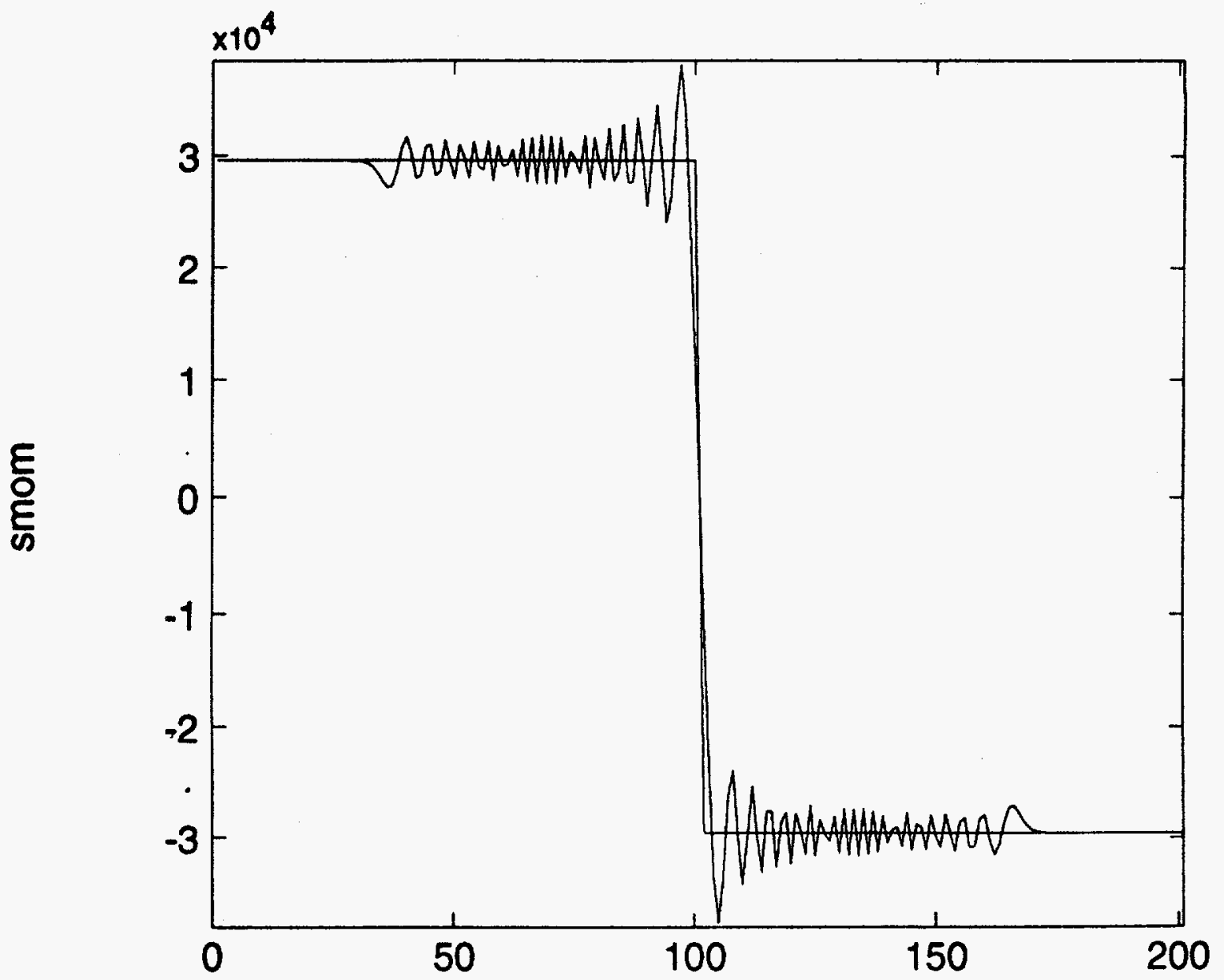

vNR SOLUTION of specific momentum at collision

Figure 6.3 


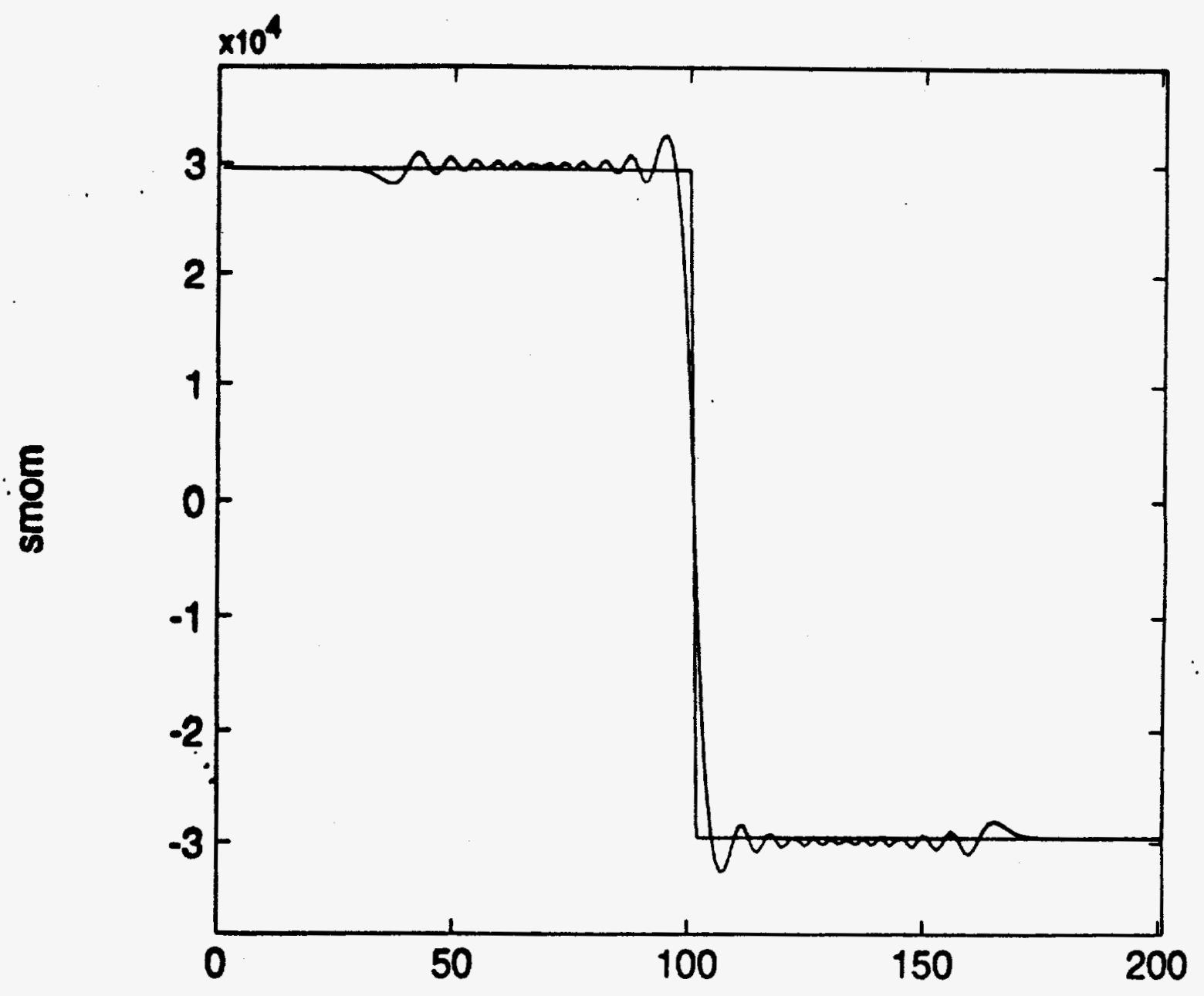

S.P.H. SOLUTION of specific momentum at collision

Figure 6.4 


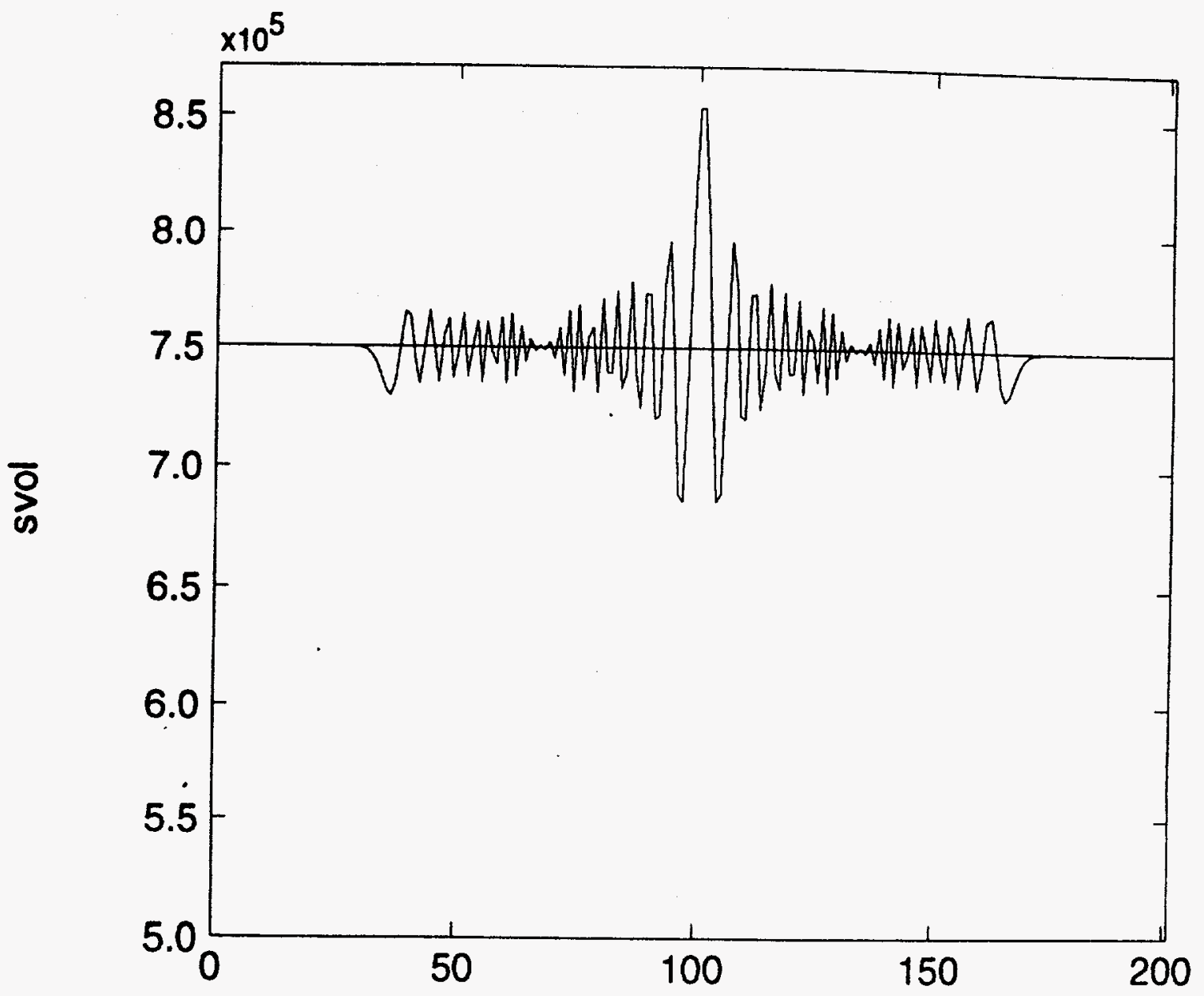

vNR SOLUTION of specific volume at collision

Figure 6.5 


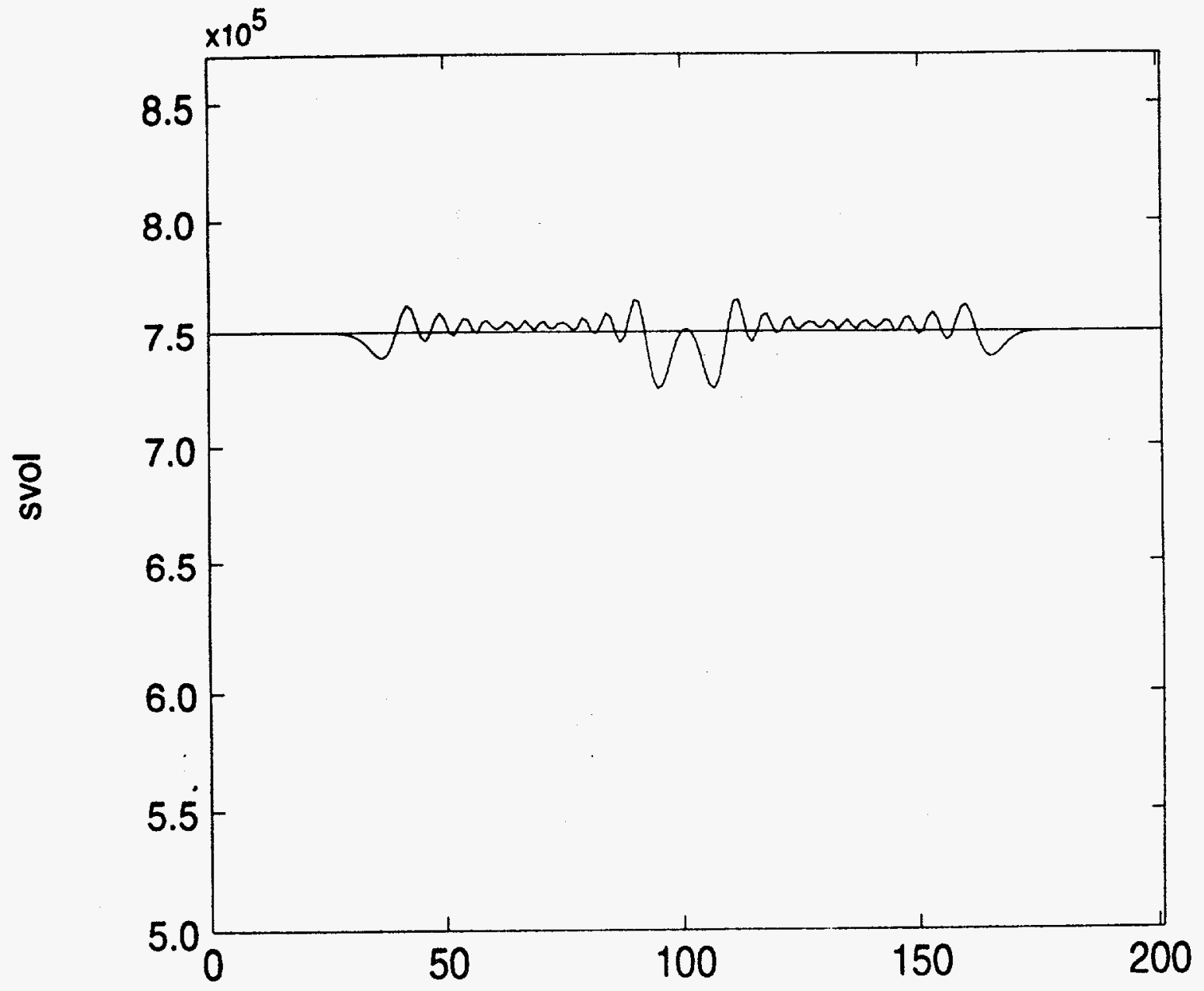

S.P.H. SOLUTION of specific volume at collision

Figure 6.6 


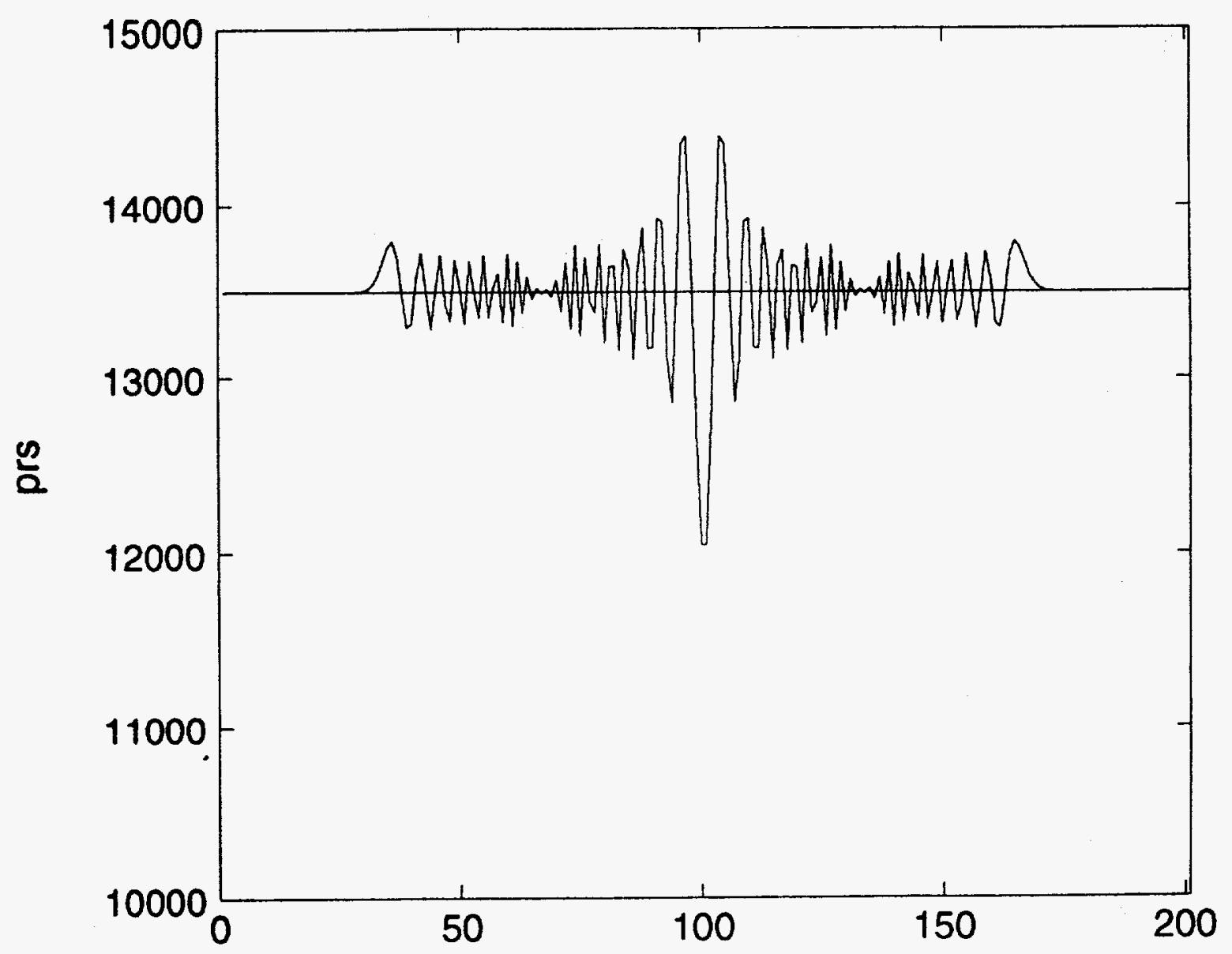

vNR SOLUTION of pressure at collision

Figure 6.7 


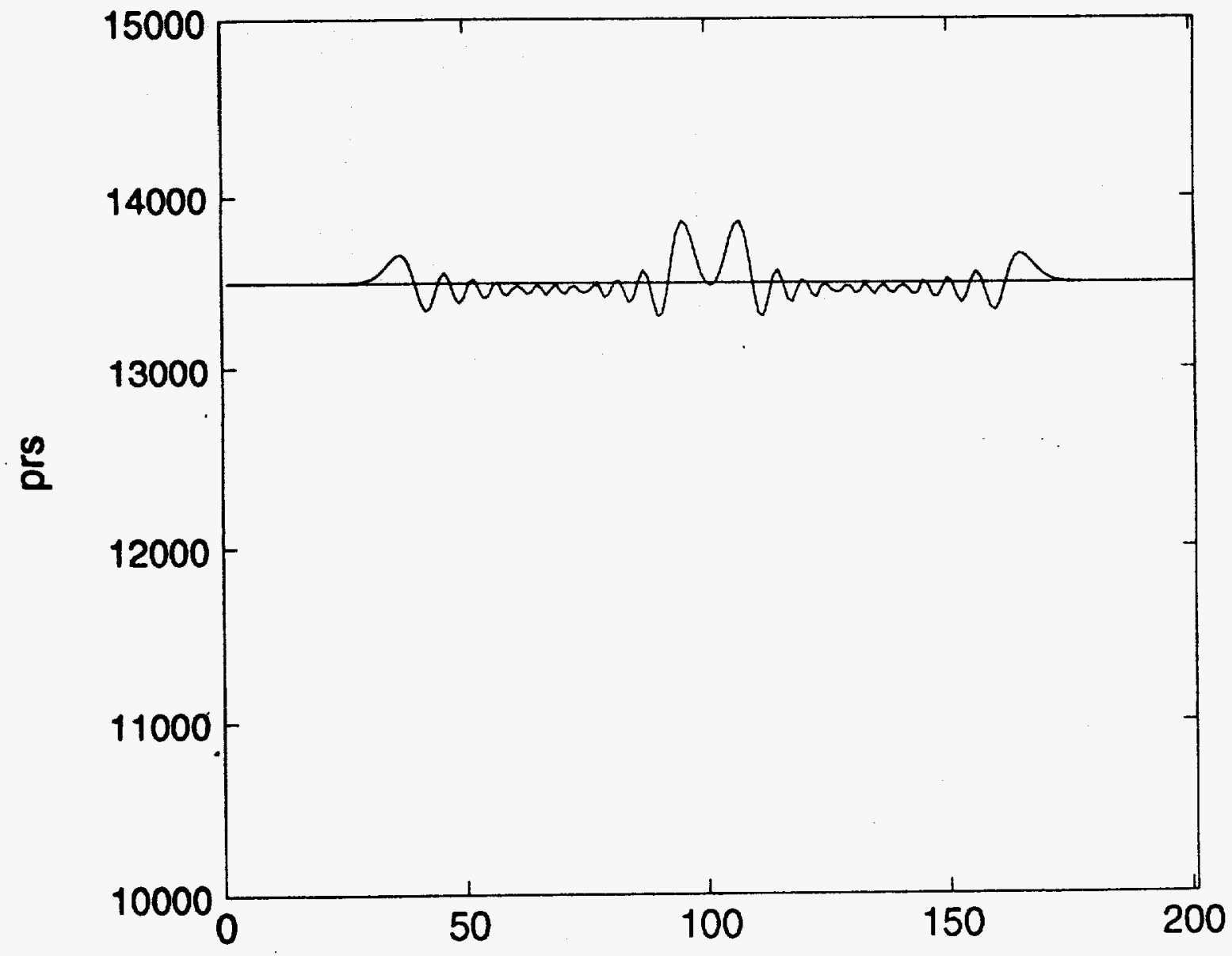

S.P.H. SOLUTION of pressure at collision

ligure 6.8 


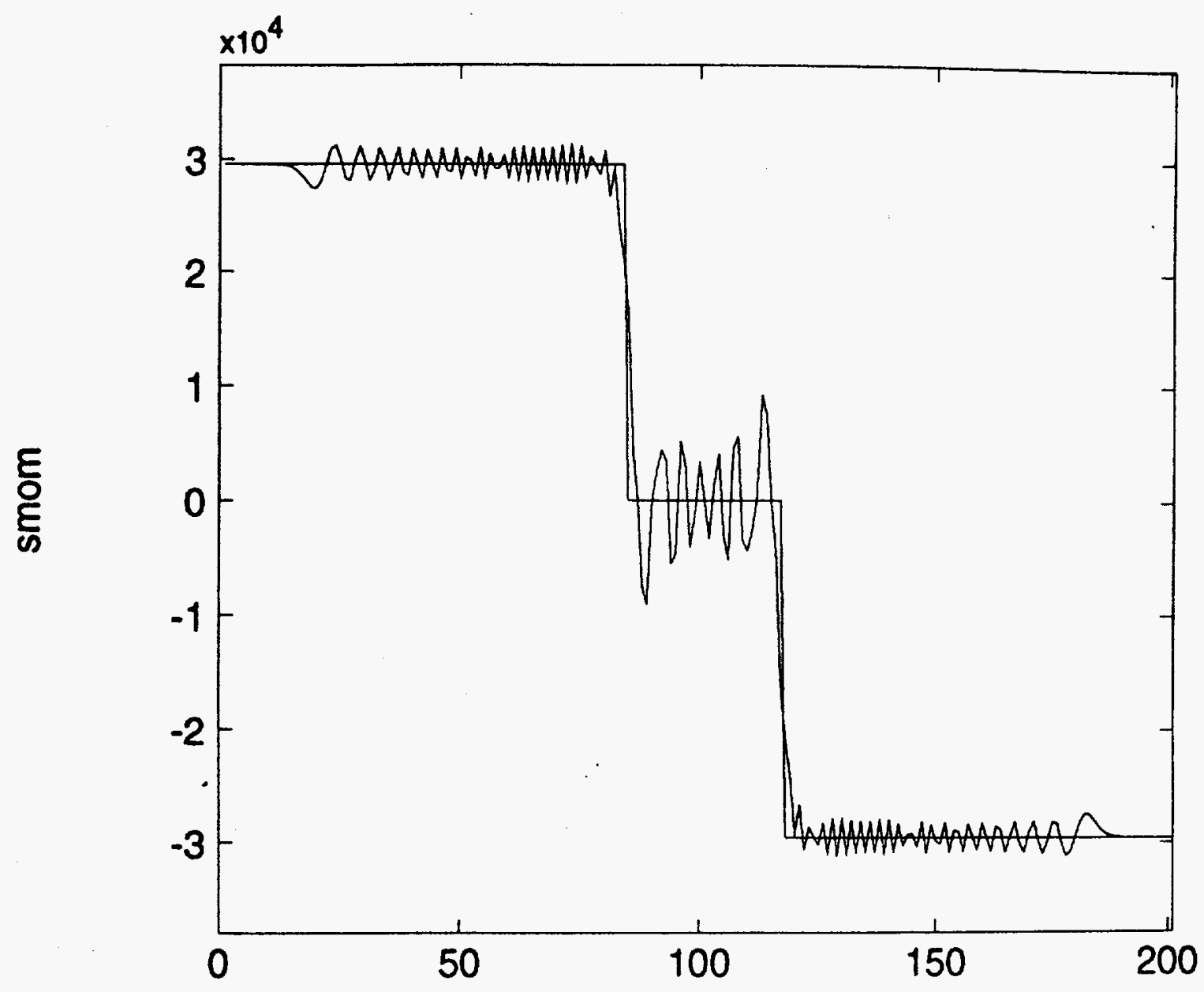

vNR SOLUTION of specific momentum after collision

Figure 6.9 


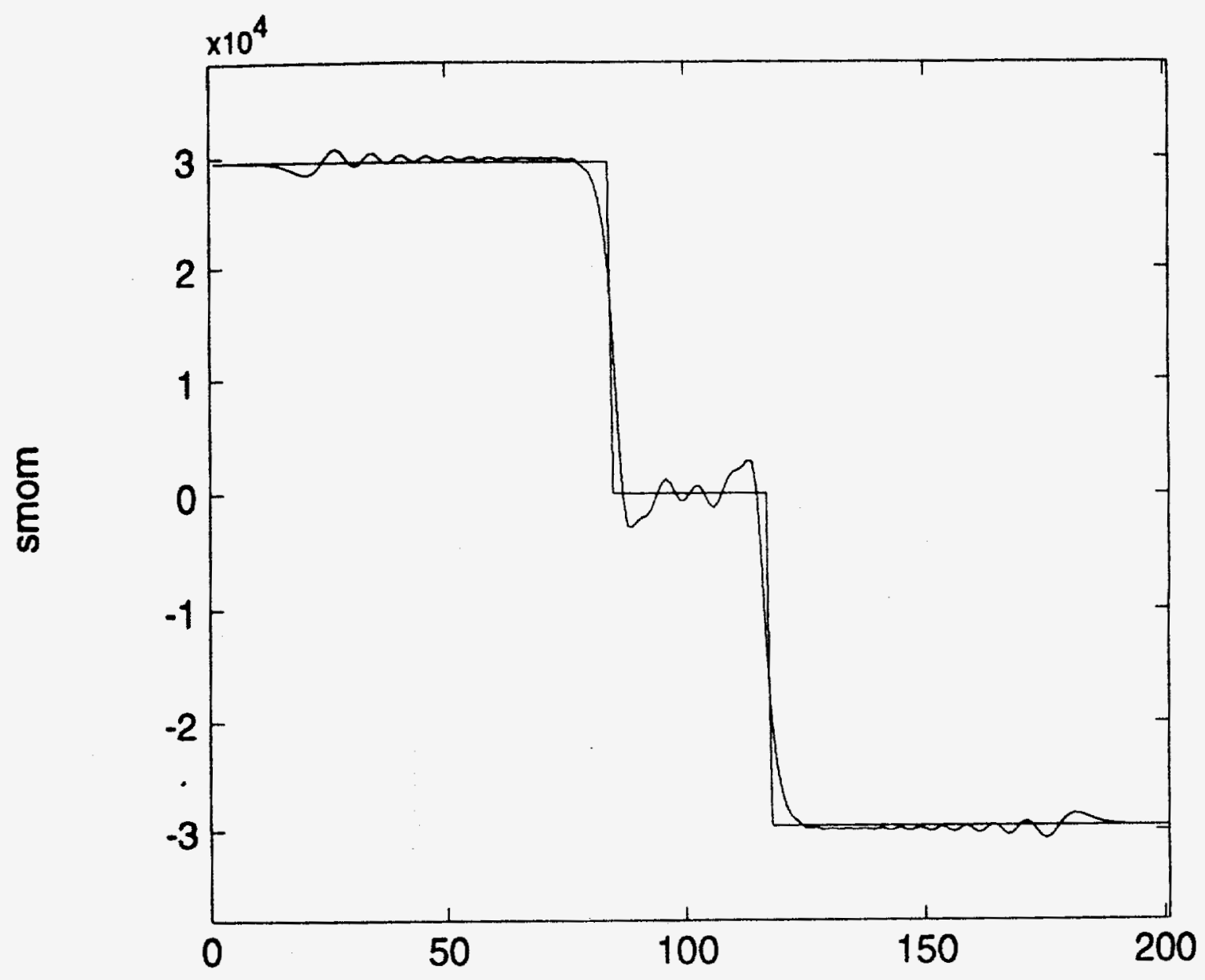

S.P.H. SOLUTION of specific momentum after collision

Figure 6.10 


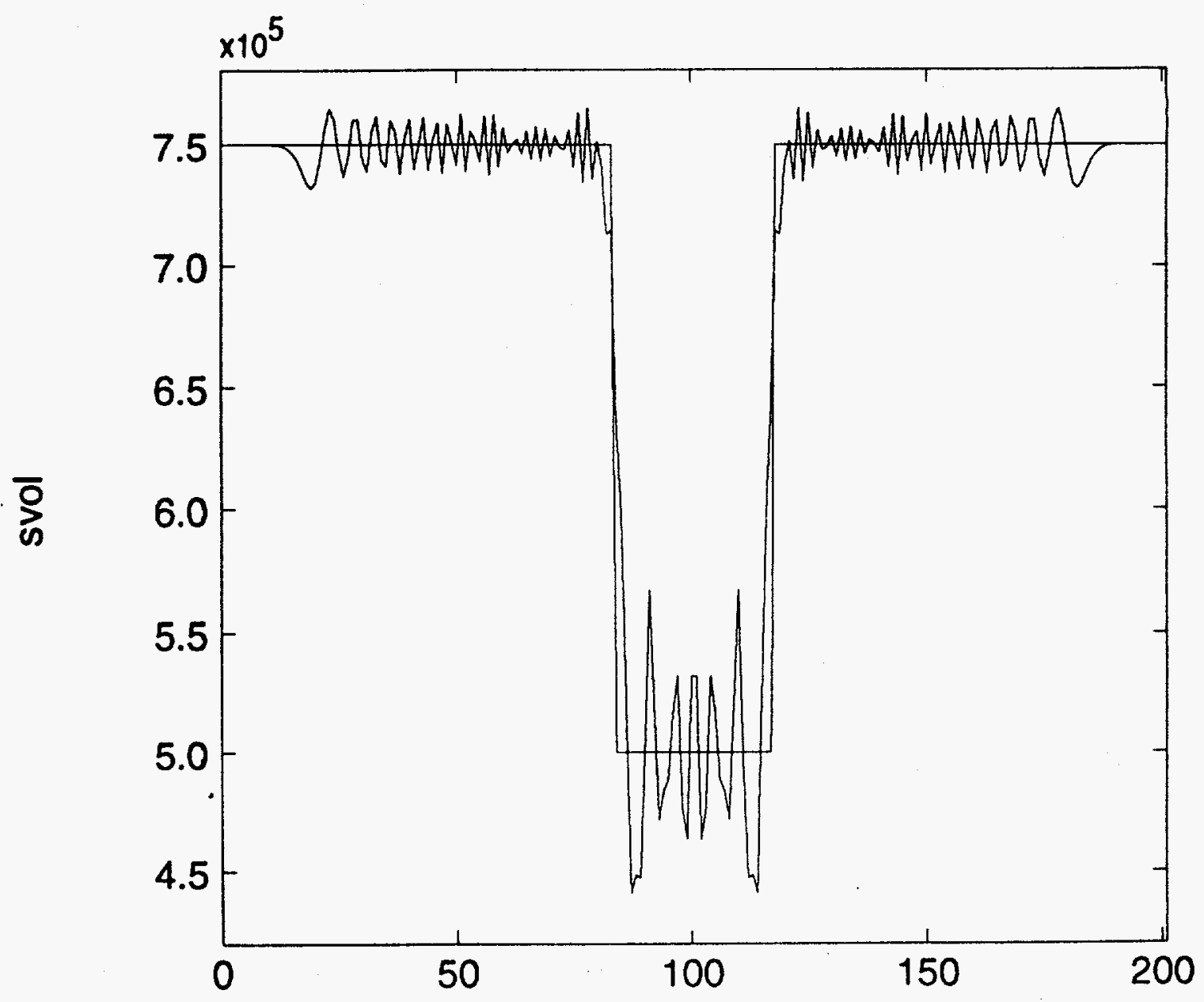

vNR SOLUTION of specific volume after collision

Figure 6.11 


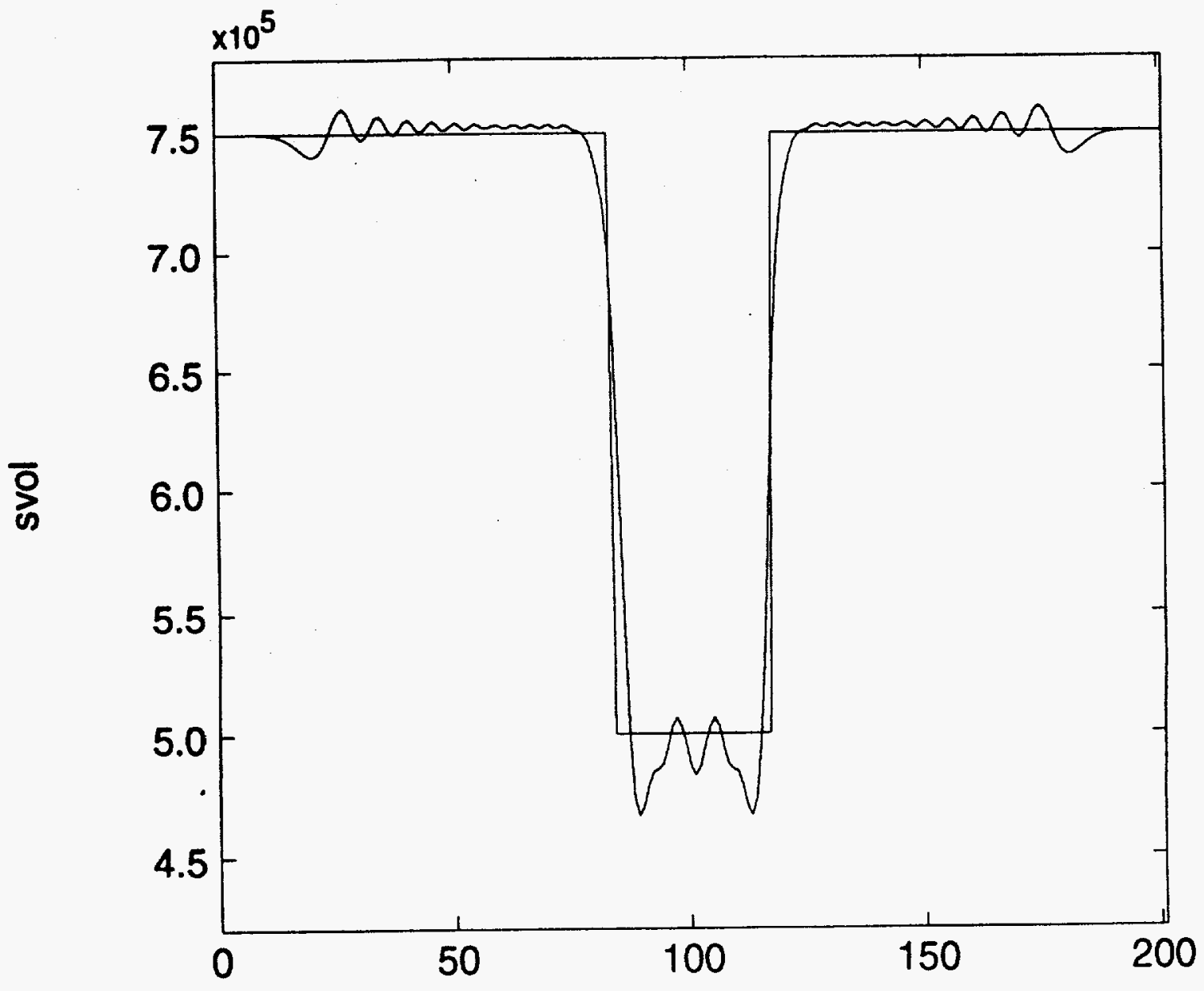

S.P.H. SOLUTION of specific volume after collision

Figure 6.12 


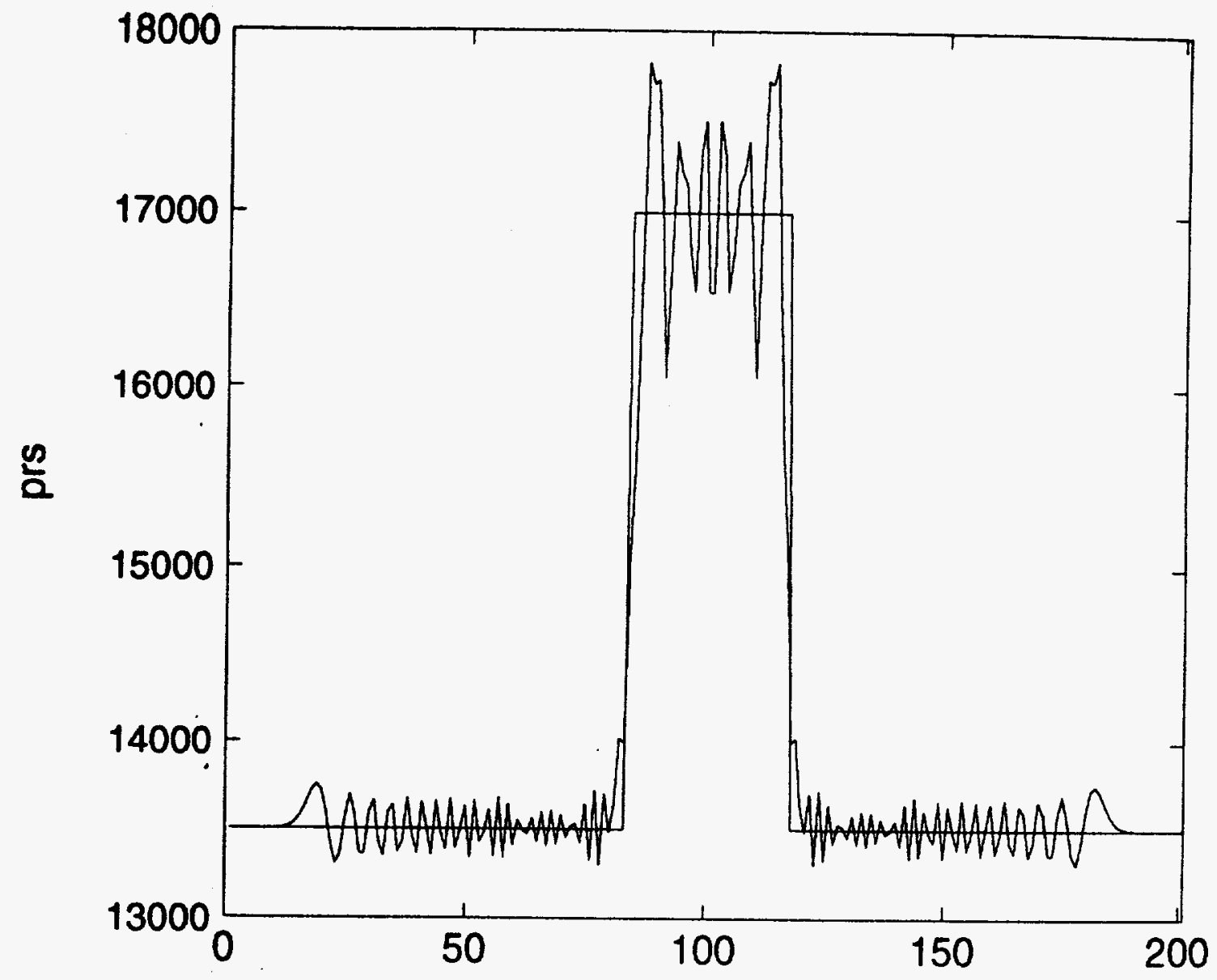

VNR SOLUTION of pressure after collision

Figure 6.13 


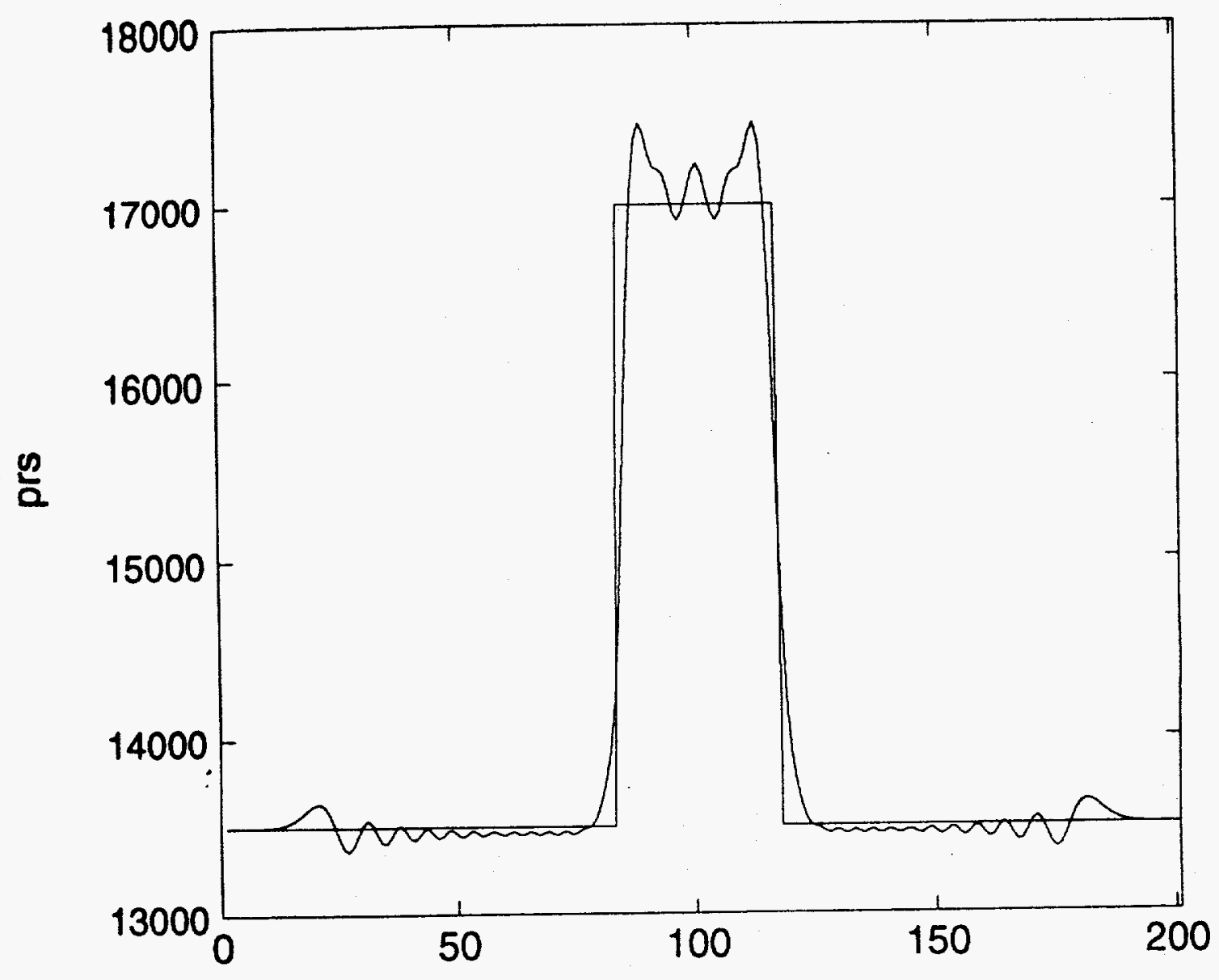

S.P.H. SOLUTION of pressure after collision

Figure 6.14 


\subsection{Summary}

The analysis in this report shows that the SPH method has an instability; this stability analysis analyzes the growth of a perturbation from a nonuniform state of stress and a uniform state of $\Delta x$. This instability is not stabilizeable with artificial viscosities. However, the analysis shows that it is stabilizeable with conservative smoothing.

The computational results in this report show that SPHCS, an SPH code with conservative smoothing, can produce more accurate solutions than $v N R$, a code based on the von Neumann - Richtmyer method, on a test problem with $C_{F L}=.2$. This is illustrated using the $v N R$ scheme on a test problem with known exact solution involving a shock collision in a Hooke's law material. 


\section{REFERENCES}

[1] J. E. Barnes, L. E. Hernquist(1993), "Computer models of colliding galaxies", Physics Today, V46, N3, pp54-61.

[2] R. Courant, K. Friedrichs, H. Lewy(1928), 'Über die partiellen Differenzengleichungen der mathematischen Physik', Math. Ann., V100, p.32.

[3] I. M. Gel'fand and G. E. Shilov (1964), Generalized Functions, Vol. I Properties and Operations, Academic Press, New York, 423pp.

[4] C. Guenther, D. L. Hicks, J.W. Swegle (1994), 'Conservative smoothing versus artificial viscosity', to be a Sandia National Laboratories Report.

[5] D. L. Hicks (1968), "Hydrocode Test Problems", Air Force Weapons Labs. Report, A.F.W.L.-T.R.-67-127, 142p.

[6] D. L. Hicks (1969), "The Convergence of Numerical Solutions of Hydrodynamic Shock Problems", Air Force Weapons Labs. Report, A.F.W.L.-T.R.-69-20, 120p.

[7] D. L. Hicks(1978), "Stability Analysis of WONDY (a Hydrocode based on the Artificial Viscosity Method of von Neumann and Richtmyer) for a Special Case of Maxwell's Material Law", Mathematics of Computation, V32, N144, p1123-1130.

[8] D. L. Hicks, J. W. Swegle, S. W. Attaway (1994), 'Conservative Smoothing Stabilizes Discrete Numerical Instabilities in S.P.H. for Solid Material Dynamics', submitted.

[9] R. Landshoff(1955), 'A numerical method for treating fluid flow in the presence of shocks,' Los Alamos National Lab. Report, LA-1930.

[10] J. von Neumann, R. Richtmyer (1950), 'A Method for the Numerical Calculation of Hydrodynamic Shocks,' J. Applied Physics, V21, N3, p232-237.

[11] R. Richtmyer, K. Morton (1967), Difference Methods for Initial Value Problems, Interscience Publishers.

[12] J. W. Swegle, D. L. Hicks, S. W. Attaway (1994), 'Stability Analysis of S.P.H.', submitted

[13] T. Tezduyar, S. Aliabadi, M. Behr, A. Johnson, S. Mittal (1993), 'Parallel Finite - Element Computation of 3D Flows', IEEE Computer, V26, N10, pp27-36

[14] Y. Wen(1994), 'Stabilizing Discrete-Numerical Instability in S.P.H. Computations with Conservative Smoothing', Masters Thesis done under the supervision of Prof. D. L. Hicks, Department of Mathematical Sciences, Michigan Technological University, 55p. 


\section{DISTRIBUTION:}

S. Atluri

Center for the Advancement of Computational Mechanics

School of Civil Engineering Georgia Institute of Technology

Atlanta, GA 30332

E. B. Becker

Department of Aerospace Eng. and Engineering Mechanics

The University of Texas at Austin

Austin, TX 78712-1085

T. Belytschko

Department of Civil Engineering

Northwestern University

Evanston, IL 60201

Dave Benson

Department of Applied Mechanics and Engineering Sciences

University of California San Diego

La Jolla, CA 92093

Naury K. Birnbaum

Century Dynamics Incorporated

7700 Edgewater Dr., Suite 626

Oakland CA 94621

Gregory Clifford

Cray Research Park

655E Lone Oak Drive

Eagan, MN 55121

Peter Cundall

ITASCA Consulting Group, Inc.

1313 Fifth Street, S.E.

Minneapolis, MN 55414
Carl Dyka

Naval Research Lab

Materials Science \& Technology

Building 28, Code 6386

4555 Overlook Avenue SW

Washington, DC 20375-5000

R. Douglas Everhart

Battelle

505 King Avenue

Columbus, OH 43201-2693

D. P. Flanagan

Hibbitt, Karlsson \& Sorrensen, Inc.

100 Medway St.

Providence, RI 02906

Arlo Fossum

RE/SPEC Inc.

Box 725

Rapid City, SD 57709

Gerry Goudreau

Methods Development Group

Mechanical Engineering Department

Lawrence Livermore National Lab

Livermore, CA 94550

Jerome B. Johnson

USACRREL

Building 4070

Ft. Wainwright, AK 99703

Sheldon Jones

Kaman Sciences

P.O. Box 7463

Colorado Springs, CO 80933-7463

David W. Keck

CONVEX Computer Corporation

P.O. Box 833851 M.S. MAR

Richardson, TX 75083-3851 
Raymond D. Krieg

Engineering Science and Mechanics

301 Perkins Hall

University of Tennessee

Knoxville, TN 37996-2030

Hans Mair, Code R14

Naval Surface Warfare Center

10901 New Hampsure Ave.

Silver Spring, MD 20903-5000

Loren K. Miller

Goodyear Technical Center

P.O. Box 3531

Akron, $\mathrm{OH}$ 44309-3531

S. Nemat-Nasser

Department of Applied Mechanics and Engineering Sciences

University of California San Diego

La Jolla, CA 92093

J. T. Oden

Department of Aerospace Eng. and Engineering Mechanics

The University of Texas at Austin

Austin, TX 78712-1085

Allan B. Pifko

Grumman Corporate Technology

Bethpage, NY 11714-3580

Mark Rashid

Department of Civil \& Environmental Engineering

University of California

Davis, CA 95616-5294

J. S. (Gus) Rice

Caterpillar Inc. Technical Center

Division 927

P.O. Box 1875

Peoria, IL 61656-1875
Steven F. Rieco

POD Associates, Inc.

2309 Renard Pl, Suite 201

Albuquerque, NM 87106

R. G. Sauvé

Mechanical Research Department

Ontario Hydro

700 University Avenue C26

Toronto, Ontario M5G 1X6

Canada

L. M. Taylor

Hibbitt, Karlsson \& Sorrensen, Inc.

100 Medway St.

Providence, RI 02906

2 Air Force Institute of Technology

Dept. of Mathematics \& Statistics

2950 P Street

Attn: Michael Stoecker

Dave Fulk

Wright-Patterson Air Force Base

Dayton, $\mathrm{OH}$ 45433-7765

Gordon Johnson

Alliant Tech Systems, Inc.

(MN 11-2925)

600 2nd St. NE

Hopkins, MN 55343

Marv Alme

Alme and Associates

2 Stevens Forest Prof. Center

9650 Santiago Road

Columbia, MD 21045

Ted Carney

Applied Research Associates

4300 San Mateo Blvd. NE

Suite A-220

Albuquerque, NM 87110 
3 Batelle

505 King Avenue

Attn: Mike Fisher

Doug Everhart

Chuck Hargraves

Columbus, OH 43201-2693

2 Cray Research, Inc.

6565 Americas Parkway, NE

Suite 830

Attn: Phil Campbell

Dave Shirley

Albuquerque, NM 87110

Lou Baker

Dagonet Software

2904 La Veta Dr. NE

Albuquerque, NM 87110-3110

Lawrence Livermore National Lab

Bill Hoover

MS L-794

P. O. Box 808

Livermore, CA 94550

15 Darrell L. Hicks

Michigan Tech. University

Math Department

Houghton, MI 49931

Joe Monaghan

Monash University

Mathematics Department

Clayton, Vic. 3168

Australia

Larry Libersky

NM Institute of Mining and Tech.

Center for Explosives Tech. Research

Socorro, NM 87801
5 Phillips Laboratory

PL/WSSD

Kirtland Air Force Base

Attn: Charles Luehr

David Medina

James Ninter

Brad Smith

Firooz Allahdadi

Albuquerque, NM 87117

Willy Benz

University of Arizona

Steward Observatory

Tucson, AZ 85721

Rita Smith

University of New Mexico

Dept. of Chemical/Nuclear Engin.

Farris Engineering Center

Albuquerque, NM 87131-1341

12 Los Alamos National Laboratory

Los Alamos, NM 87545

Attn:

J. P. Hill, WX-11, MS C931

B. L. Holian, T-12, MS B268

D. A. Rabern, MEE-4, MS G787

P. S. Follansbee, MS G756

D. Mandell, X-3, MS F663

R. F. Davidson, N-6, MS K557

N. L. Johnson, T-3, MS B261

J. K. Dienes, N-6, MS K557

C. A. Anderson, MS J576

M. W. Lewis, MEE-4, MS G787

C. Wingate, MS F645

B. Stellingwerf, MS F645 
Sandia Internal:

MS1187 1271 George Allshouse

MS1184 1239 Frank Dempsey

MS0321 1400 Ed Barsis

MS0441 1425 Johnny Biffle

MS0441 1425 Stephen Attaway

MS0441 1425 Mark Blanford

MS0441 1425 Marilyn Smith

MS0820 1433 M.E. Kipp

MS0439 1434 David Martinez

MS0841 1500 D. J. McCloskey

MS0836 1501 C. W. Peterson

MS0827 1502 P. J. Hommert

MS0827 1511 J. S. Rottler

MS0827 1511 Jim Schutt

MS0834 1512 A. C. Ratzel

MS0835 1513 R. D. Skocypec

MS0832 1551 W. P. Wolfe

MS0833 1552 C. E. Hailey

MS0826 1553 W. L. Hermina

MS0825 1554 W. H. Rutledge

16 MS0443 1561 H. S. Morgan \& Staff

14 MS0437 1562 R. K. Thomas \& Staff

25 MS0437 1562 J. W. Swegle

MS0336 1707 Kim Mahin

MS0515 2561 S.T. Montgomery

MS0660 2861 Randall Lober

MS0457 5600 Dennis Hayes

MS0574 5941 John Schamaun

MS0724 6000 Dan Hartley

MS0751 6117 Dale Preece

MS1325 6313 Joseph Jung

MS1143 6500 James Rice

MS1145 6514 Jim Fisk

MS1145 6514 Joel Miller

MS9214 8117 William Mason

MS9401 8702 Bill Robinson

MS9043 8743 George Johnson

MS9042 8741 Juanita Benson

MS9042 8741 Michael Chiesa

MS9042 8742 Jay Dike

MS9042 8742 Paul Jin

MS9042 8742 Bruce Kistler

MS9042 8742 Khanh Trinh

MS9043 8743 Melvin Callabresi
MS9043 8743 Douglas Bammann

MS9043 8743 Lee Bertram

MS9043 8743 Mark Horstemeyer

MS9043 8743 James Lathrop

MS9043 8743 Arthur Ortega

MS9043 8743 Vincent Prantil

MS9043 8745 William Winters

MS0974 9421 Jeanne Ramage

5 MS0899 7141 Technical Library

MS0619 7151 Tech Publications

10 MS01007613-2 Document Processing for DOE/OSTI

MS90188523 Central Tech Files 